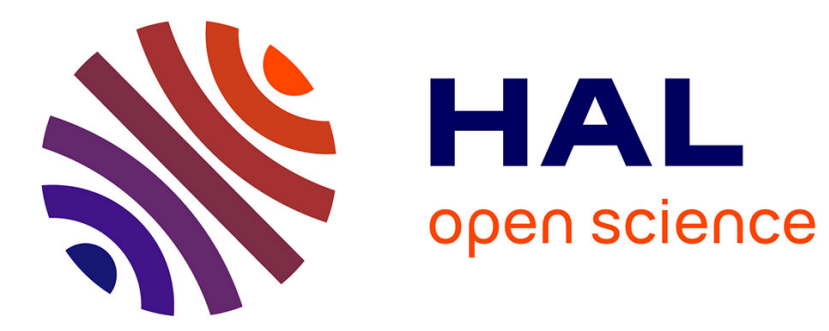

\title{
Estimation for stochastic differential equations with mixed effects.
}

\author{
Valentine Genon-Catalot, Catherine Larédo
}

\section{To cite this version:}

Valentine Genon-Catalot, Catherine Larédo. Estimation for stochastic differential equations with mixed effects.. Statistics, 2016, 50 (5), pp.1014-1035. hal-00807258v3

\section{HAL Id: hal-00807258 \\ https://hal.science/hal-00807258v3}

Submitted on 7 Dec 2015

HAL is a multi-disciplinary open access archive for the deposit and dissemination of scientific research documents, whether they are published or not. The documents may come from teaching and research institutions in France or abroad, or from public or private research centers.
L'archive ouverte pluridisciplinaire HAL, est destinée au dépôt et à la diffusion de documents scientifiques de niveau recherche, publiés ou non, émanant des établissements d'enseignement et de recherche français ou étrangers, des laboratoires publics ou privés. 


\title{
ESTIMATION FOR STOCHASTIC DIFFERENTIAL EQUATIONS WITH MIXED EFFECTS.
}

\author{
V. GENON-CATALOT ${ }^{1}$ AND C. LARÉDO ${ }^{2}$
}

\begin{abstract}
We consider the long term behaviour of a one-dimensional mixed effects diffusion process $(X(t))$ with a multivariate random effect $\phi$ in the drift coefficient. We first study the estimation of the random variable $\phi$ based on the observation of one sample path on the time interval $[0, T]$ as $T$ tends to infinity. The process $(X(t))$ is not Markov and we characterize its invariant distributions. We build moments and maximum likelihood-type estimators of the random variable $\phi$ which are consistent and asymptotically mixed normal with rate $\sqrt{T}$. Moreover, we obtain non asymptotic bounds for the moments of these estimators. Examples with a bivariate random effect are detailed. Afterwards, the estimation of parameters in the distribution of the random effect from $N$ i.i.d. processes $\left(X_{j}(t), t \in[0, T]\right), j=1, \ldots, N$ is investigated. Estimators are built and studied as both $N$ and $T=T(N)$ tend to infinity. We prove that the convergence rate of estimators differs when deterministic components are present in the random effects. For true random effects, the rate of convergence is $\sqrt{N}$ whereas for deterministic components, the rate is $\sqrt{N T}$. Illustrative examples are given.
\end{abstract}

AMS 2000 subject classification: Primary: 62M05, 62F12 ; secondary: 60J60.

Keywords and phrases: Asymptotic mixed normality, invariant distributions, mixed-effects stochastic differential equations, parametric inference, random effects estimation.

\section{Running title: Mixed effects estimation for SDEs.}

\section{INTRODUCTION}

Stochastic differential equations constitute a well-established tool for modelling physical phenomena whose dynamics are affected by random noise. Some situations require a hierarchical modelling of the dynamics of $X$, with first a random variable $\phi$ (the random effect) and second, given that $\phi=\varphi$, an SDE with drift and diffusion coefficient depending on $(x, \varphi)$ that rules the $X($.$) 's dynamics. This occurs in particular when it is of interest to model simultaneously$ the performance of several experiments or when adding this hierarchical modelling is needed to estimate population parameters. Usually, $X$ represents the behaviour of an individual, the random components of $\phi$ describe the individual specificity and, given $\phi=\varphi$, the model describes a general evolution for all individuals with individual value $\varphi$ (see e.g. Davidian and Giltinan (1995), Pinheiro and Bates (2000), Kuhn and Lavielle (2004), Nie and Yang (2005), Nie (2006), Nie (2007)). Stochastic differential equations (SDE) with mixed effects have been introduced to generalize classical ordinary differential equations models ( Ditlevsen and De Gaetano (2005), Overgaard et al. (2005), Donnet and Samson (2008)) and also to model neuronal data (Picchini et al. (2010)).

The continuous-time stochastic process $X=(X(t), t \geq 0)$ with dynamics ruled by a stochastic differential equation with mixed effects satisfies

$$
d X(t)=b(X(t), \phi) d t+\sigma(X(t), \phi) d W(t), \quad X(0)=\eta,
$$

${ }^{1}$ Université Paris Descartes, MAP5, UMR CNRS 8145.

${ }^{2}$ Laboratoire MaIAGE, I.N.R.A. and LPMA, Université Denis Diderot, CNRS-UMR 7599. 
where $W$ is a Brownian motion, $\phi$ is a random variable with distribution $\nu(d \varphi),(\phi, \eta)$ is a random variable independent of $W$.

Recently, the estimation of the unknown distribution $\nu(d \varphi)$ of $\phi$ whether parametric or nonparametric based on the observation of $N$ i.i.d. processes $\left.\left(X_{j}(t), t \in[0, T]\right), j=1, \ldots, N\right)$ distributed as (1.1) has been the subject of several contributions. Parametric estimation is investigated in Ditlevsen and De Gaetano (2005) for the specific model of mixed effects Brownian motion with drift and in Donnet and Samson (2008). In Delattre et al. (2012), the maximum likelihood estimator (MLE) for random effects SDEs is studied more generally for fixed $T$ and $N$ tending to infinity (see also Maitra and Bhattacharya (2014) for non i.i.d. sample paths). In particular, for $\phi \in \mathbb{R}$ having a Gaussian distribution with unknown mean $\mu$ and unknown variance $\omega^{2}, b(x, \varphi)=\varphi b(x), \sigma(x, \varphi)=\sigma(x)$ and $b(),. \sigma($.$) known functions, an explicit formula for the$ exact likelihood is obtained and a complete asymptotic study of the exact MLE of $\left(\mu, \omega^{2}\right)$ is given. Approximations of the likelihood are also proposed for general mixed SDEs in Picchini et al. (2010). For $\phi$ real-valued and $\nu(d \varphi)=f(\varphi) d \varphi$, nonparametric estimation of the density $f$ is investigated in Comte et al. (2013), under the asymptotic framework that both $N$ and $T=T(N)$ tend to infinity in such a way that $T(N) / N$ tends to infinity. Only two specific models are studied: $b(x, \varphi)=\varphi b(x), b(x, \varphi)=\varphi+b(x), \sigma(x, \varphi)=\sigma(x)$. Nonparametric adaptive estimation of a mixed-effect $\phi$ in the drift coefficient of an Ornstein-Uhlenbeck process has been investigated in Dion (2014). The general case of mixed-effects both in the drift and diffusion coefficient of multidimensional diffusions is also addressed from a practical view point in Picchini and Ditlevsen (2011) with implementation of estimators. For an SDE with a linear mixed effect in the diffusion coefficient, the estimation of population parameters is investigated in Delattre et al. (2014).

Our focus here is first to study the long term behaviour of a one-dimensional mixed effects SDE,

$$
d X(t)=b(X(t), \phi) d t+\sigma(X(t)) d W(t), \quad X(0)=\eta
$$

where $\sigma(x, \varphi)=\sigma(x)$ is a known real-valued function defined on $\mathbb{R}, W$ is a standard Wiener process and $(\phi, \eta)$ is a random variable independent of $W$ taking values in $\Phi \times \mathbb{R}$ with $\Phi \subset \mathbb{R}^{d}$. We study the properties of model (1.2). The process $(X(t))$ is not Markov and has infinitely many invariant distributions when $\phi$ is random. We are interested in the estimation of the random variable $\phi$ based on the observation of one trajectory $(X(t), t \in[0, T])$ as $T$ tends to infinity. Let us point out that it is not a statistical problem in the usual sense since $\phi$ is not a parameter but a random variable. Nevertheless, several recent papers now deal with the estimation of random variables. One of the most popular examples concerns the estimation of the stochastic integrated volatility in semimartingales models based on high frequency observations of the sample path (see Kessler et al. (2012), Chapters 2 and 3). To build estimators of $\phi$, two methods are investigated, a moment type method and a maximum likelihood approach. Both lead to estimators which are consistent and asymptotically mixed normal at rate $\sqrt{T}$. These two methods are applied on classical examples: the Ornstein-Uhlenbeck and the C.I.R. processes with a bivariate random effect.

Second, we investigate the framework where $N$ i.i.d. processes $\left(X_{j}(t), t \in[0, T], j=1, \ldots, N\right)$ are observed and each sample path $X_{j}$ is associated with a random effect $\phi_{j}$. We are interested in estimating unknown parameters in the distribution $\nu(d \varphi)$ of the $\phi_{j}$ 's (the population parameters). The results of the first part lead to consider a two-stage procedure. First, build for each $j$, an estimator of $\phi_{j}$ from the trajectory $\left(X_{j}(t), t \leq T\right)$. Then, use a plug-in technique to derive estimators of unknown parameters of $\nu(d \varphi)$ and study their asymptotic properties as both $N$ and $T$ tend to infinity. Using moment type estimators, we prove that the convergence rate of estimators differs when deterministic components (fixed effects) are present in the $\phi_{j}$ 's. For true 
random effects, the rate of convergence is $\sqrt{N}$ whereas for deterministic components, the rate is $\sqrt{N T}$. This is consistent with Nie's results (Nie (2007)). The case of the Ornstein-Uhlenbeck process with two random effects is especially detailed, i.e. the observed processes are given by $d X_{j}(t)=-\phi_{2, j}\left(X_{j}(t)-\phi_{1, j}\right) d t+d W_{j}(t), X_{j}(0)=\eta_{j}$ where $\phi_{j}=\left(\phi_{1, j}, \phi_{2, j}\right) \in \mathbb{R} \times(0,+\infty)$. Estimators of $\mathbb{E} \phi_{1}$ and $\mathbb{E} \phi_{2}^{-1}$ are studied both when the two components of $\phi_{j}$ are random or when only one is random while the other one is deterministic. The C.I.R. process (square root diffusion) with two random effects is also studied.

Next, we choose a specific model to develop a maximum likelihood type method. We concentrate on the Ornstein-Uhlenbeck model with one multiplicative random effect in the drift, i.e. $d X_{j}(t)=-\phi_{j} X_{j}(t) d t+d W_{j}(t), X_{j}(0)=\eta_{j}$ where $\phi_{j} \in(0,+\infty)$. Assuming that $\phi_{j}$ follows a Gamma distribution with unknown parameters $(a, \lambda)$, we define appropriate estimators of $\phi_{j}$ and $\log \phi_{j}$ that we plug in the likelihood function associated with i.i.d. Gamma random variables. This defines a contrast. We prove that, as both $N, T=T(N)$ tend to infinity, the associated maximum contrast estimator is asymptotically equivalent to the maximum likelihood estimator based on the observation of $\left(\phi_{j}, j=1, \ldots, N\right)$ provided that $N / T$ tends to 0 .

The paper is organized as follows. Section 2 contains notations, assumptions and results on the invariant distributions of (1.2). Section 3 is concerned with the estimation of the random effect $\phi$ when only one sample path is continuously observed on $[0, T]$ as $T \rightarrow \infty$. Section 3.1 is devoted to moment type estimators. Classical theorems for ergodic diffusions with no random effects are interpreted and restated for the case of diffusions with random effects. The maximum likelihood type estimators are presented in Section 3.2. In Section 4, we consider that observations are $N$ i.i.d. trajectories on $[0, T]$ and study the estimation of unknown parameters in the distribution of random effects (the population parameters). Section 5 contains extensions and concluding remarks. Proofs are gathered in Section 6.

\section{NotATIONS, ASSUMPTIONS AND PRELIMINARY RESUlT.}

Consider a real valued stochastic process $(X(t), t \geq 0)$, with dynamics ruled by (1.2). The Wiener process $W$ and the r.v. $(\phi, \eta)$ are defined on a common probability space $(\Omega, \mathcal{F}, \mathbb{P})$ endowed with a filtration $\left(\mathcal{F}_{t}, t \geq 0\right)$ satisfying the usual conditions. The couple $(\phi, \eta)$ is assumed $\mathcal{F}_{0}$-measurable and $W$ is a $\left(\mathcal{F}_{t}, t \geq 0\right)$-Brownian motion. We use the classical notations for random effects models, i.e. $\phi$ for the random variable and $\varphi$ for the fixed value. We denote by $X^{\varphi, x_{0}}$ the process solution of the SDE with fixed $\varphi$ and initial condition $x_{0}$ :

$$
d X^{\varphi, x_{0}}(t)=b\left(X^{\varphi, x_{0}}(t), \varphi\right) d t+\sigma\left(X^{\varphi, x_{0}}(t)\right) d W(t), \quad X^{\varphi, x_{0}}(0)=x_{0}
$$

We introduce now assumptions on model (1.2).

[H1] The real valued function $(x, \varphi) \rightarrow b(x, \varphi)$ (resp. $x \rightarrow \sigma(x)$ ) is continuous on $\mathbb{R} \times \mathbb{R}^{d}$ (resp. $\mathbb{R})$ and such that the process $(\phi, X(t))$ where $X(t)$ is given by (1.2) is well defined and adapted to the filtration $\left(\mathcal{F}_{t}\right)$ for all initial condition $(\phi, \eta)$.

[H2] (i) There exists an open subset $\Phi$ of $\mathbb{R}^{d}$ and an interval $(\ell, r) \subset \mathbb{R}$ such that $\forall x \in$ $(\ell, r), \sigma^{2}(x)>0$, and $\forall \varphi \in \Phi$, the scale density of $(2.1)$

$$
s_{\varphi}(x)=\exp \left(-2 \int_{x_{0}}^{x} \frac{b(u, \varphi)}{\sigma^{2}(u)} d u\right), \quad x_{0} \in(\ell, r)
$$

satisfies $\int_{\ell} s_{\varphi}(x) d x=\int^{r} s_{\varphi}(x) d x=+\infty$ and the speed density $m_{\varphi}(x)=1 /\left(\sigma^{2}(x) s_{\varphi}(x)\right)$ of $(2.1)$ satisfies $M(\varphi):=\int_{\ell}^{r} m_{\varphi}(x) d x<+\infty$.

[H2] (ii) The function $(x, \varphi) \rightarrow b(x, \varphi)$ is $C^{1}$ on $(\ell, r) \times \mathbb{R}^{d}$ and $x \rightarrow \sigma(x)$ is $C^{2}$ on $(\ell, r)$.

[H2] (iii) The random variable $\phi$ takes values in $\Phi$. We denote its distribution by $\nu(d \varphi)$. 
[H3] The random couple $(\phi, \eta)$ has distribution $\pi^{\nu}(d \varphi, d x)=\nu(d \varphi) \otimes \pi_{\varphi}(x) d x$ where

$$
\pi_{\varphi}(x)=1_{(\ell, r)}(x) \frac{m_{\varphi}(x)}{M(\varphi)} .
$$

Standard regularity assumptions on $b(x, \varphi)$ and $\sigma(x)$ ensure (H1) (see e.g. Karatzas and Shreve (2000) for general results and Comte et al. (2013) for the specific context of SDEs with mixed effects). Under $[\mathbf{H 1}]$, the process $(\phi, X(t))$ is strong Markov. The Markov property is enlighted by looking at (1.2) as a system:

$$
\begin{aligned}
d \phi(t) & =0, \quad \phi(0)=\phi \\
d X(t) & =b(X(t), \phi(t)) d t+\sigma(X(t)) d W(t), \quad X(0)=\eta
\end{aligned}
$$

Thus, $(\phi, X(t))$ is a two dimensional diffusion process with initial distribution

$$
\mu^{\nu}(d \varphi, d x)=\nu(d \varphi) \otimes \mu_{\varphi}(d x)
$$

where $\nu$ is the distribution of $\phi$ and $\mu_{\varphi}$ is a distribution on $(\ell, r)$ corresponding to the conditional distribution of $\eta$ given $\phi=\varphi$. Assumption [H2] is classical for existence and uniqueness of invariant distributions for SDEs with non random parameters (see e.g. Kessler et al. (2012)). The unique invariant density of model $(2.1)$ is $(2.2)$. Denote by $p_{t}^{\varphi}(x, y)$ the transition density of model (2.1). Assumptions [H1]-[H2] imply the measurability of

$$
(x, y, \varphi) \rightarrow p_{t}^{\varphi}(x, y) \quad \text { and } \quad(x, \varphi) \rightarrow \pi_{\varphi}(x) .
$$

For $g:(\ell, r) \rightarrow \mathbb{R}$ a positive or $\pi_{\varphi}$-integrable Borel function, $\varphi \rightarrow \pi_{\varphi}(g):=\int_{\ell}^{r} g(x) \pi_{\varphi}(x) d x$ is measurable on $\Phi$. Therefore, we can define the random variables

$$
\pi_{\phi}(g)=\int_{\ell}^{r} g(x) \pi_{\phi}(x) d x \quad \text { and the sets }
$$

$$
L^{1}(\Phi)=\left\{g:(\ell, r) \rightarrow \mathbb{R} \text {, Borel } \forall \varphi \in \Phi, \pi_{\varphi}(|g|)<+\infty\right\}
$$

$$
L^{2}(\Phi)=\left\{g:(\ell, r) \rightarrow \mathbb{R}, \text { Borel } \forall \varphi \in \Phi, \pi_{\varphi}\left(g^{2}\right)<+\infty\right\} .
$$

For $f \in C^{2}((\ell, r))$, let us introduce the infinitesimal generator of $(2.1)$ :

$$
\mathcal{L}_{\varphi} f(x)=\frac{1}{2} \sigma^{2}(x) f^{\prime \prime}(x)+b(x, \varphi) f^{\prime}(x)=\frac{1}{2 m_{\varphi}(x)}\left(\frac{f^{\prime}}{s_{\varphi}}\right)^{\prime}(x) .
$$

To $g \in L^{2}(\Phi)$, we associate a function $F_{\varphi, g}$ such that $(x, \varphi) \rightarrow F_{\varphi, g}(x) \in C^{2}((\ell, r) \times \Phi)$, for all $\varphi \in \Phi, \pi_{\varphi} F_{\varphi, g}^{2}<+\infty$, the limit of $F_{\varphi, g}^{\prime} / s_{\varphi}$ as $x \rightarrow \ell, r$ is null, and

$$
\mathcal{L}_{\varphi} F_{\varphi, g}=-\left(g-\pi_{\varphi}(g)\right) \text {. }
$$

From (2.8), we have $F_{\varphi, g}^{\prime}(x)=-2 s_{\varphi}(x) \int_{\ell}^{x}\left(g(u)-\pi_{\varphi}(g)\right) m_{\varphi}(u) d u$. If moreover $g$ satisfies

$$
\int_{\ell}^{r}\left(F_{\varphi, g}^{\prime}(x) \sigma(x)\right)^{2} \pi_{\varphi}(x) d x:=V_{\varphi}(g)<+\infty,
$$

then, for $<., .>_{\varphi}$ denoting the scalar product of $L^{2}\left(\pi_{\varphi}\right)$,

$$
-2<\mathcal{L}_{\varphi} F_{\varphi, g}, F_{\varphi, g}>_{\varphi}=V_{\varphi}(g),
$$


(see Genon-Catalot et al. (2000)) for details on the existence of such function $F_{\varphi, g}$ and (2.9)(2.11). In the case of two functions $g, h$ such that $V_{\varphi}(g)<+\infty, V_{\varphi}(h)<+\infty$, we set

$$
V_{\varphi}(g)=V_{\varphi}(g, g) \quad \text { and } \quad V_{\varphi}(g, h)=\int_{\ell}^{r} F_{\varphi, g}^{\prime}(x) F_{\varphi, h}^{\prime}(x) \sigma^{2}(x) \pi_{\varphi}(x) d x .
$$

Later on (in Sections 3 and 4), we consider the following condition for $g \in L^{2}(\Phi)$ and $F_{\varphi, g}$ defined by (2.9) satisfying (2.10) for all $\varphi \in \Phi$ :

- $(\mathbf{K} \gamma)$ : For $\gamma \geq 1$ and for all $\varphi \in \Phi$,

$$
K_{\gamma}(\varphi, g):=\pi_{\varphi}\left(F_{\varphi, g}^{2 \gamma}\right)+\pi_{\varphi}\left(\left(F_{\varphi, g}^{\prime} \sigma\right)^{2 \gamma}\right)<+\infty
$$

This technical condition is used to obtain non asymptotic bounds for the moments estimators defined below. It can be checked easily on classical models.

Proposition 2.1. Assume $[\mathbf{H 1}]$ and consider the process $(\phi, X(t))$ given by (1.2) where the initial variable $(\phi, \eta)$ has distribution $\mu^{\nu}$ (see (2.3)) with $\nu$ a distribution on $\Phi$ and $\mu_{\varphi}$ a distribution on $(\ell, r)$. Then, given that $\phi=\varphi$, the conditional distribution of $(X(t))$ is identical to the distribution of $X^{\varphi}$ given by

$$
d X^{\varphi}(t)=b\left(X^{\varphi}(t), \varphi\right) d t+\sigma\left(X^{\varphi}(t)\right) d W(t), \quad X^{\varphi}(0) \sim \mu_{\varphi}, X^{\varphi}(0) \quad \text { independent of } W .
$$

Assume [H1]-[H2]. Then, the distribution $\pi^{\nu}(d \varphi, d x)=\nu(d \varphi) \otimes \pi_{\varphi}(x) d x$ on $\Phi \times(\ell, r)$ is an invariant distribution for the Markov process $(\phi, X(t))$.

The proof of Proposition 2.1 is easily obtained by comparing the finite-dimensional distributions and using that, by the Markov property of $(\phi,(X(t))$, the conditional distribution of $(X(t))$ given $\phi=\varphi, X(0)=x_{0}$ is identical to the distribution of $X^{\varphi, x_{0}}$ satisfying $(2.1)$. When $(\phi, \eta)$ has distribution $\pi^{\nu}$ (assumption [H3]), the process $(\phi, X(t))$ is strictly stationary with marginal distribution $\pi^{\nu}$. The Markov process $(\phi, X(t))$ having infinitely many invariant distributions is not ergodic. The marginal distribution of $X(0)$ when $(\phi, X(0))$ has distribution $\pi^{\nu}$ is a mixture distribution with density given by $p^{\nu}(x)=\int_{\Phi} \nu(d \varphi) \pi_{\varphi}(x)$. The conditional distribution of $X$ given $\phi=\varphi$ is the distribution of the stationary and ergodic solution of the SDE with fixed $\varphi$. Below, we provide general results and apply them on examples. Certain developments can be detailed only in specific examples. We introduce now the models on which we rely to illustrate the estimation methods proposed here.

\section{Examples 2.1.}

Model 1. Ornstein-Uhlenbeck process with one random effect:

$$
d X(t)=-\phi X(t) d t+d W(t), \quad X(0)=\eta
$$

with $\phi \in \Phi=(0,+\infty)$. The invariant distribution of the model with fixed $\varphi$ is $\pi_{\varphi}(d x)=$ $\mathcal{N}(0,1 /(2 \varphi))(d x)$.

Model 2. Ornstein-Uhlenbeck process with two random effects:

$$
d X(t)=-\phi_{2}\left(X(t)-\phi_{1}\right) d t+d W(t), X(0)=\eta .
$$

With $\phi=\left(\phi_{1}, \phi_{2}\right) \in \Phi=\mathbb{R} \times(0,+\infty)$, the invariant distribution of the model with fixed $\varphi$ is $\pi_{\varphi}(d x)=\mathcal{N}\left(\varphi_{1}, 1 /\left(2 \varphi_{2}\right)\right)(d x)$.

Model 3. C.I.R. process with two random effects:

$$
d X(t)=-\phi_{2}\left(X(t)-\phi_{1}\right) d t+\sqrt{X(t)} d W(t), X(0)=\eta .
$$

Applying [H2], we take $\phi=\left(\phi_{1}, \phi_{2}\right) \in \Phi=\left\{2 \varphi_{1} \varphi_{2} \geq 1, \varphi_{2}>0\right\}$. We have $\pi_{\varphi}=G\left(2 \varphi_{1} \varphi_{2}, 2 \varphi_{2}\right)$ the Gamma distribution with tail parameter $2 \varphi_{1} \varphi_{2}$ and scale parameter $2 \varphi_{2}$. 


\section{Estimation of A RANDOM EFFECT IN THE DRIFT.}

We investigate two methods to estimate the random variable $\phi$ based on the observation of one trajectory $(X(t), t \in[0, T])$ in the asymptotic framework $T \rightarrow+\infty$. For this, we need to extend some classical limit theorems for ergodic diffusions with no random effects to the case of diffusions with random effects.

3.1. Moments method. We consider the estimation of the random variable $\pi_{\phi}(g)$ (see (2.5)) by

$$
\widetilde{\pi_{\phi}(g)}=\frac{1}{T} \int_{0}^{T} g(X(s)) d s .
$$

For a vector of functions $\underline{g}=\left(g_{1}, \ldots, g_{k}\right)^{\prime}$, we define the vector

$$
\widetilde{\pi_{\phi}(\underline{g})}=\left(\frac{1}{T} \int_{0}^{T} g_{i}(X(s)) d s, i=1, \ldots, k\right)^{\prime} .
$$

Proposition 3.1. Assume $[\mathbf{H 1}]-[\mathbf{H 2}]$ and consider the process $(\phi, X(t))$ defined in (1.2) where the initial variable $(\phi, \eta)$ has distribution $\mu^{\nu}$ (see (2.3)). For $g \in L^{1}(\Phi)$, almost surely, as $T$ tends to infinity,

$$
\widetilde{\pi_{\phi}(g)} \rightarrow \pi_{\phi}(g)
$$

For the following two propositions, we consider $g, g_{1}, \ldots, g_{k} \in L^{2}(\Phi)$ and let $F_{\varphi, g}, F_{\varphi, g_{i}}, i=$ $1, \ldots k$ be defined by $(2.9)$ and satisfy $(2.10)$ for all $\varphi \in \Phi$.

Proposition 3.2. Assume $[\mathbf{H 1}]-[\mathbf{H 2}]$ and consider the process $(\phi, X(t))$ given by (1.2) where the initial variable $(\phi, \eta)$ has distribution $\mu^{\nu}$ (see (2.3)). Then,

$\sqrt{T}\left(\widetilde{\pi_{\phi}(g)}-\pi_{\phi}(g)\right)$ converges stably in law to the mixed normal law $M \mathcal{N}\left(0, V_{\phi}(g)\right)$, i.e. the law of $\varepsilon\left(V_{\phi}(g)\right)^{1 / 2}$ with $\varepsilon \sim \mathcal{N}(0,1)$ independent of $V_{\phi}(g)$.

Assume that the matrix $V_{\varphi}(\underline{g}):=\left(V_{\varphi}\left(g_{i}, g_{j}\right)\right)_{1 \leq i, j \leq k}$ is invertible for all $\varphi \in \Phi$ (see (2.12)). Then, the random vector

$$
\left.\sqrt{T} \widetilde{\left(\pi_{\phi}(\underline{g})\right.}-\pi_{\phi}(\underline{g})\right)
$$

converges stably in law to the mixed normal law $M \mathcal{N}_{k}\left(0, V_{\phi}(\underline{g})\right)$.

From now on, we assume that $[\mathbf{H 1}]-[\mathbf{H 2}]-[\mathbf{H 3}]$ hold i.e. that the process $(X(t))$ is in stationary regime. We thus consider $(X(t))$ be given by $(1.2)$ where the initial variable $(\phi, \eta)$ has distribution $\pi^{\nu}$ with $\pi_{\varphi}$ defined in (2.2) (stationary regime). Note that conditionally on $\phi$, $(X(t))$ is also stationary.

The previous results (Propositions 3.1-3.2) show that the random variable $\pi_{\phi}(g)$ for a given function $g$ is consistently estimated by $\widetilde{\pi_{\phi}(g)}$. As the process is in stationary regime, we have $\mathbb{E}(g(X(s)) \mid \phi)=\pi_{\phi}(g)$ for all $s$, so the estimators are conditionally unbiased:

$$
\mathbb{E}\left(\widetilde{\pi_{\phi}(g)} \mid \phi\right)=\pi_{\phi}(g) \text {. }
$$

Define, using (2.10):

$$
\rho(g, \phi)=2 \pi_{\phi}\left(F_{\phi, g}^{2}\right)+2\left(2 V_{\phi}(g) \pi_{\phi}\left(F_{\phi, g}^{2}\right)\right)^{1 / 2} .
$$


ESTIMATION FOR STOCHASTIC DIFFERENTIAL EQUATIONS WITH MIXED EFFECTS. 7

Proposition 3.3. Assume $[\mathbf{H 1}]-[\mathbf{H 2}]-[\mathbf{H 3}]$. For g satisfying condition $(\mathbf{K} \gamma)$,

$$
\begin{gathered}
\left.\mathbb{E}\left(\left[\sqrt{T} \widetilde{\pi_{\phi}(g)}-\pi_{\phi}(g)\right)\right]^{2 \gamma} \mid \phi\right) \leq c_{\gamma} K_{\gamma}(\phi, g), \\
\left.\mathbb{E}\left(\left[\sqrt{T} \widetilde{\left(\pi_{\phi}(g)\right.}-\pi_{\phi}(g)\right)\right]^{2} \mid \phi\right)=V_{\phi}(g)+Z_{T}(g, \phi),
\end{gathered}
$$

where $c_{\gamma}$ is a numerical constant and $\left|Z_{T}(g, \phi)\right| \leq \frac{1}{\sqrt{T}} \rho(g, \phi)$.

For two functions $g, h$ such that $V_{\varphi}(g)<+\infty, V_{\varphi}(h)<+\infty$ for all $\varphi \in \Phi$, we have

$$
\left.\left.\mathbb{E}\left(\left[T \widetilde{\left(\pi_{\phi}(g)\right.}-\pi_{\phi}(g)\right) \widetilde{\left(\pi_{\phi}(h)\right.}-\pi_{\phi}(h)\right)\right] \mid \phi\right)=V_{\phi}(g, h)+Z_{T}(g, h, \phi),
$$

where $\left|Z_{T}(g, h, \phi)\right| \leq \frac{1}{\sqrt{T}}(\rho(g+h, \phi)+\rho(g-h, \phi))$.

Remark 3.1. Note that the upper bounds for $Z_{T}(g, \phi), Z_{T}(g, h, \phi)$ can be improved on examples (see below) where these quantities are found of order $1 / T$ by exact computation.

We illustrate the method on examples.

\section{Examples 3.1.}

Model 2 (continued). Consider Model 2 (see 2.1), the Ornstein-Uhlenbeck process with two random effects. Assume that $(\phi, \eta)$ has distribution $\nu(d \varphi) \otimes \pi_{\varphi}(d x)$ with $\nu$ a distribution on $\Phi$. Define the estimators of $\phi_{1}, \tau=\phi_{2}^{-1}, \phi_{2}$ by

$$
\widetilde{\phi}_{1}=\frac{1}{T} \int_{0}^{T} X(s) d s, \quad \widetilde{\tau}=2\left(\frac{1}{T} \int_{0}^{T} X^{2}(s) d s-\left(\widetilde{\phi}_{1}\right)^{2}\right), \quad \widetilde{\phi}_{2}=\frac{1}{\widetilde{\tau}} .
$$

Note that $\tau$ is a natural parameter here as it is proportional to the variance of $\pi_{\varphi}$.

As T tends to infinity, $\left(\widetilde{\phi}_{1}, \widetilde{\phi}_{2}\right)$ converges a.s. to $\left(\phi_{1}, \phi_{2}\right)$ and $\sqrt{T}\left(\widetilde{\phi}_{1}-\phi_{1}, \widetilde{\phi}_{2}-\phi_{2}\right)^{\prime}$ is asymptotically $\mathcal{M N}_{2}(0, J(\phi))$ where

$$
J(\phi)=\left(\begin{array}{cc}
\phi_{2}^{-2} & 0 \\
0 & 2 \phi_{2}
\end{array}\right) .
$$

Note that, if $\phi_{2}$ is deterministic, so is $J(\phi)$ and the asymptotic distribution is Gaussian. On the contrary, if $\phi_{1}=m$ is deterministic, $\sqrt{T}\left(\widetilde{\phi}_{1}-m\right)$ is still asymptotically mixed normal $\mathcal{M N}\left(0, \phi_{2}^{-2}\right)$.

For the conditional moments, the following holds ( $\lesssim$ means $\leq$ up to a constant):

$$
\begin{gathered}
\mathbb{E}\left(\left(\widetilde{\phi}_{1}-\phi_{1}\right)^{2} \mid \phi\right)=\frac{\tau^{2}}{T}+Z_{T}, \quad\left|Z_{T}\right| \leq \frac{\tau^{3}}{T^{2}} . \\
\mathbb{E}\left(\left(\widetilde{\phi}_{1}-\phi_{1}\right)^{2 \gamma} \mid \phi\right) \lesssim T^{-\gamma}\left(\tau^{3 \gamma}+\tau^{2 \gamma}\right), \\
\mathbb{E}(\widetilde{\tau}-\tau \mid \phi)=-\frac{2 \tau^{2}}{T}+\frac{2 \tau^{3}\left(1-e^{-T / \tau}\right)}{T^{2}}, \quad \mathbb{E}\left((\widetilde{\tau}-\tau)^{2} \mid \phi\right)=\frac{2 \tau^{3}}{T}+\zeta_{T}, \\
\mathbb{E}\left((\widetilde{\tau}-\tau)^{2 \gamma} \mid \phi\right) \lesssim T^{-\gamma}\left(\tau^{2 \gamma}+\tau^{3 \gamma}+\tau^{4 \gamma}+\tau^{6 \gamma}+\phi_{1}^{2 \gamma} \tau^{3 \gamma}+\phi_{1}^{2 \gamma} \tau^{2 \gamma}\right),
\end{gathered}
$$

where $\left|\zeta_{T}\right| \leq \frac{1}{T \sqrt{T}} C(\phi)$ and $C(\phi)$ depends on powers of $\phi_{1}, \tau$. Note that $\widetilde{\tau}$ is not conditionally unbiased. If $\mathbb{E}\left(\tau^{3 \gamma}+\tau^{2 \gamma}\right)<+\infty$, we have $\left.\mathbb{E}\left(\widetilde{\phi}_{1}-\phi_{1}\right)^{2 \gamma}\right) \leq C T^{-\gamma}$. Thus, the constraint only depends on the distribution of $\phi_{2}$. If the r.h.s. of (3.12) has a finite expectation, $\mathbb{E}(\widetilde{\tau}-\tau)^{2 \gamma} \leq$ $C T^{-\gamma}$. 
Model 3 (continued). Consider now Model 3 (see 2.1), the C.I.R. process process with two random effects. Assume that $(\phi, \eta)$ has distribution $\nu(d \varphi) \otimes \pi_{\varphi}(d x)$ for $\nu$ a distribution on $\Phi$. Consider the estimators of $\phi_{1}, \beta=\phi_{1} /\left(2 \phi_{2}\right), \phi_{2}$ given by

$$
\widetilde{\phi}_{1}=\frac{1}{T} \int_{0}^{T} X(s) d s, \quad \widetilde{\beta}=\frac{1}{T} \int_{0}^{T} X^{2}(s) d s-\left(\widetilde{\phi}_{1}\right)^{2}, \quad \widetilde{\phi}_{2}=\frac{\widetilde{\phi}_{1}}{2 \widetilde{\beta}} .
$$

Then, as $T$ tends to infinity, $\left(\widetilde{\phi}_{1}, \widetilde{\phi}_{2}\right)$ converges a.s. to $\left(\phi_{1}, \phi_{2}\right)$ and $\sqrt{T}\left(\widetilde{\phi}_{1}-\phi_{1}, \widetilde{\phi}_{2}-\phi_{2}\right)^{\prime}$ is asymptotically $\mathcal{M N}_{2}\left(0, J_{\text {cir }}(\phi)\right)$ where

$$
J_{\text {cir }}(\phi)=\left(\begin{array}{cc}
\phi_{1} \phi_{2}^{-2} & -\phi_{2}^{-1} \\
-\phi_{2}^{-1} & 4 \phi_{2}+2 \phi_{1}^{-1}
\end{array}\right) .
$$

For the conditional moments, the following holds, for $\gamma \geq 1$, :

$$
\begin{gathered}
\mathbb{E}\left(\left(\widetilde{\phi}_{1}-\phi_{1}\right)^{2} \mid \phi\right)=\frac{\phi_{1}}{T \phi_{2}^{2}}+Z_{T}, \quad\left|Z_{T}\right| \leq \frac{\phi_{1}}{T^{2} \phi_{2}^{3}}, \\
\mathbb{E}\left(\left(\widetilde{\phi}_{1}-\phi_{1}\right)^{2 \gamma} \mid \phi\right) \lesssim T^{-\gamma} \phi_{2}^{-2 \gamma}\left(\phi_{1}^{\gamma}+\phi_{1}^{2 \gamma}\right) .
\end{gathered}
$$

Even if either $\phi_{1}$ or $\phi_{2}$ is deterministic, $J_{\text {cir }}(\phi)$ is still stochastic. Note that, as $2 \phi_{1} \phi_{2} \geq 1$, $\phi_{1} \phi_{2}^{-3} \leq 2 \phi_{1}^{2} \phi_{2}^{-2}$. Thus, (3.13) and (3.14) are not contradictory. The conditional moments of $\widetilde{\beta}$ could also be obtained with additional computations.

(Proof in Appendix).

3.2. Maximum likelihood method. We can also use a maximum likelihood approach.

3.2.1. General drift. Assumption [H2] implies that $\mathbb{P}(\forall s \geq 0, \sigma(X(s))>0 \mid \phi=\varphi)=1$ for all $\varphi \in \Phi$. Therefore, $\mathbb{P}(\forall s \geq 0, \sigma(X(s))>0)=1$ and the function

$$
L_{T}(\psi)=\exp \left(\int_{0}^{T} \frac{b(X(s), \psi)}{\sigma^{2}(X(s))} d X(s)-(1 / 2) \int_{0}^{T} \frac{b^{2}(X(s), \psi)}{\sigma^{2}(X(s))} d s\right)
$$

is well-defined for all $\psi \in \Phi$. Thus, we can introduce:

$$
\hat{\phi}_{T}=\hat{\phi}_{T}(X(s), s \leq T)=\operatorname{Argsup}_{\psi} L_{T}(\psi) .
$$

The functional $\hat{\varphi}_{T}=\hat{\phi}_{T}\left(X^{\varphi, x_{0}}(s), s \leq T\right)$ is the exact maximum likelihood of the true value $\varphi$ based on the observation $\left(X^{\varphi, x_{0}}(s), s \leq T\right)$. Consider the additional assumptions:

[H4] The function $(x, \varphi) \rightarrow b(x, \varphi)$ is twice continuously differentiable on $(\ell, r) \times \mathbb{R}^{d}$.

[H5] The following matrix $I(\varphi)=\left(I_{j k}(\varphi)\right)$ is invertible for $\varphi \in \Phi$ :

$$
I_{j k}(\varphi)=\int_{(\ell, r)} \frac{\left(\partial b / \partial \varphi_{j}\right)(x, \varphi)\left(\partial b / \partial \varphi_{k}\right)(x, \varphi)}{\sigma^{2}(x)} \pi_{\varphi}(x) d x .
$$

Proposition 3.4. Assume $[\mathbf{H 1}]-[\mathbf{H 5}]$, that the maximum likelihood estimator $\hat{\varphi}_{T}$ based on the observation $\left(X^{\varphi, x_{0}}(s), s \leq T\right)$ with fixed $\varphi$ is consistent and that $\sqrt{T}\left(\hat{\varphi}_{T}-\varphi\right)$ converges in distribution to $\mathcal{N}\left(0, I^{-1}(\varphi)\right)$ for all $\varphi \in \Phi$. Then, $\hat{\phi}_{T}$ converges in probability to $\phi$ and $\sqrt{T}\left(\hat{\phi}_{T}-\phi\right)$ converges in distribution to the mixed normal law $\mathcal{M N}\left(0, I^{-1}(\phi)\right)$.

The consistency and the asymptotic normality of the estimator $\hat{\varphi}_{T}$ is detailed e.g. in Kutoyants (2004). The proof of Proposition 3.4 is obtained by conditioning and omitted.

To illustrate Proposition 3.4, consider a multidimensional linear random effect in the drift, 
ESTIMATION FOR STOCHASTIC DIFFERENTIAL EQUATIONS WITH MIXED EFFECTS. 9

$b(., \varphi)=\sum_{i=1}^{d} \varphi^{j} b_{j}($.$) with \varphi=\left(\varphi^{j}, j=1, \ldots, d\right) \in \mathbb{R}^{d}$ and where the $b_{j}$ 's are $C^{1}(\mathbb{R})$ functions. Define the vector

$$
U_{T}=\left(\int_{0}^{T} \frac{b_{1}(X(s))}{\sigma^{2}(X(s))} d X(s) \quad \ldots \quad \int_{0}^{T} \frac{b_{d}(X(s))}{\sigma^{2}(X(s))} d X(s)\right)^{\prime}
$$

and the matrix $\mathcal{I}_{T}=\left(\mathcal{I}_{T}^{k, \ell}\right)_{1 \leq k, \ell \leq d}$ with $\mathcal{I}_{T}^{k, l}=\int_{0}^{T} \frac{b_{k}(X(s)) b_{\ell}(X(s))}{\sigma^{2}(X(s))} d s$.

The estimator $\hat{\phi}_{T}$ of $\phi$ is defined by the equation: $\mathcal{I}_{T} \hat{\phi}_{T}=U_{T}$.

Assume that, for $k=1, \ldots, d, \sigma^{-1} b_{k}$ belongs to $L^{2}(\Phi)$ (see $\left.(2.7)\right)$ and $\operatorname{set} \mathcal{I}(\phi)=\left(\pi_{\phi}\left(b_{k} b_{\ell} / \sigma^{2}\right)\right)$. Then, $\mathbb{P}\left(\mathcal{I}_{T} / T \rightarrow \mathcal{I}(\phi)\right)=1$. Denote by $\Phi_{0}$ the subset of $\Phi$ such that $\mathcal{I}(\varphi)$ invertible for all $\varphi \in \Phi_{0}$. On $\Phi_{0}, \sqrt{T}\left(\hat{\phi}_{T}-\phi\right)$ converges in distribution to $\mathcal{M N}\left(0, \mathcal{I}(\phi)^{-1}\right)$.

\section{Examples 3.2.}

Model 2 (continued). In particular, for the Ornstein-Uhlenbeck model ( see 2.1, model 2), set $\psi_{1}=\phi_{1} \phi_{2}, \psi_{2}=\phi_{2}, b_{1}(x)=1, b_{2}(x)=-x, \phi=F(\psi)=\left(\left(\psi_{1} / \psi_{2}\right) \psi_{2}\right)^{\prime}$. The computation of $\mathcal{I}(\psi)$ and $J(\phi)=D F(\psi) \mathcal{I}^{-1}(\psi)(D F(\psi))^{\prime}$ with $\psi=F^{-1}(\phi)$ yields

$$
J(\phi)=\left(\begin{array}{cc}
\phi_{2}^{-2} & 0 \\
0 & 2 \phi_{2}
\end{array}\right) .
$$

Thus, $\sqrt{T}\left(\hat{\phi}_{T}-\phi\right)$ is asymptotically $\mathcal{M N}(0, J(\phi))$ with the same matrix $J(\phi)$ as obtained in Examples 3.1.

3.2.2. Truncation technique. For Section 4, we need non asymptotic bounds for the moments of estimators of $\phi$. The exact moments of $\hat{\phi}_{T}$ are untractable. So we present a truncation technique which allows to study these moments. We detail it on the example of one linear random effect in the drift. Let $b(., \varphi)=\varphi b($.$) with \varphi \in \Phi \subset \mathbb{R}$ with $b \in C^{1}(\mathbb{R})$. The estimator of $\phi$ is:

$$
\hat{\phi}_{T}=\frac{\int_{0}^{T} \frac{b(X(s))}{\left.\sigma^{2}(X(s))\right)} d X(s)}{\int_{0}^{T} \frac{b^{2}(X(s))}{\sigma^{2}(X(s))} d s} .
$$

To obtain simple bounds, we introduce a truncated version of $\hat{\phi}_{T}$ (see (3.16)) and define, for $k$ a constant:

$$
\hat{\phi}_{T}^{(k)}=\hat{\phi}_{T} 1_{\left(\frac{V_{T}}{T} \geq \frac{k}{\sqrt{T}}\right)} \quad \text { where } \quad V_{T}=\int_{0}^{T} h(X(s)) d s, \quad h(x)=\frac{b^{2}(x)}{\sigma^{2}(x)} .
$$

Note that the event $\left(\frac{V_{T}}{T}<\frac{k}{\sqrt{T}}\right)$ has small probability (see below) and we can prove that the two estimators $\hat{\phi}_{T}, \hat{\phi}_{T}^{(k)}$ are asymptotically equivalent in the sense that $\sqrt{T}\left(\hat{\phi}_{T}-\hat{\phi}_{T}^{(k)}\right)=o_{P}(1)$.

Proposition 3.5. Assume [H1] - [H3]. Assume that, for $\gamma \geq 1$, for all $\varphi \in \Phi, \pi_{\varphi} h^{2 \gamma}<+\infty$. Assume that $F_{\varphi, h}$ defined in (2.9) satisfies Condition $(\mathbf{K} 2 \gamma)$. Then,

$$
\mathbb{E}\left(\left(\hat{\phi}_{T}^{(k)}-\phi\right)^{2 \gamma} \mid \phi\right) \leq T^{-\gamma} C(\gamma, \phi),
$$

where

$$
C(\gamma, \phi) \lesssim \frac{1}{\left[\pi_{\phi}(h)\right]^{2 \gamma}}\left(\pi_{\phi}\left(h^{\gamma}\right)+\left(\pi_{\phi}\left(h^{2 \gamma}\right) K_{2 \gamma}(\phi, h)\right)^{1 / 2}+\phi^{2 \gamma}\left(1+K_{\gamma}(\phi, h)\right)\right)
$$


Moreover, for all $p \geq 1$,

$$
\mathbb{P}\left(\frac{V_{T}}{T}<\frac{k}{\sqrt{T}} \mid \phi\right) \lesssim T^{-p}\left(\pi_{\phi} h\right)^{-2 p}\left(K_{p}(\phi, h)+1\right) .
$$

The interest of Proposition 3.5 is that it provides an explicit and non asymptotic bound for conditional moments of $\hat{\phi}_{T}^{(k)}-\phi$. By taking expectation of both sides, this provides a clear link between the unconditional moments of $\hat{\phi}_{T}^{(k)}-\phi$.

We can illustrate the above conditions for the Ornstein-Uhlenbeck process.

\section{Examples 3.3.}

Model 1 (see 2.1.) Consider the Ornstein-Uhlenbeck process with one random effect $d X(t)=$ $-\phi X(t) d t+d W(t), \quad X(0)=\eta$, with $\phi \in(0,+\infty)$ and $(\phi, \eta) \sim d \nu(\varphi) \otimes \mathcal{N}\left(0,(2 \varphi)^{-1}\right)$ where $\nu$ is a distribution on $(0,+\infty)$. Then, for all $\gamma \geq 1$,

$$
\mathbb{E}\left(\left(\hat{\phi}_{T}^{(k)}-\phi\right)^{2 \gamma} \mid \phi\right) \lesssim T^{-\gamma}\left(1+\phi^{\gamma}+\phi^{4 \gamma}+\phi^{-2 \gamma}+\phi^{-3 \gamma}\right)
$$

with $\hat{\phi}_{T}^{(k)}$ defined by (3.16)-(3.17) with $b(x)=-x, \sigma(x)=1, h(x)=x^{2}$.

We can also consider the moment type estimator of $\phi^{-1}$. For $h(x)=x^{2}$, we have $\pi_{\phi}(h)=(2 \phi)^{-1}$, $F_{\varphi, h}(x)=(2 \varphi)^{-1}\left(x^{2}-(2 \varphi)^{-1}\right)$, and we easily obtain:

$$
K_{\gamma}(\phi, h) \lesssim \phi^{-3 \gamma}+\phi^{-4 \gamma}
$$

The random variable $\phi^{-1}$ admits the conditionally unbiased estimator $(2 / T) \int_{0}^{T} X^{2}(s) d s$ and:

$$
\mathbb{E}\left(\left(\frac{2}{T} \int_{0}^{T} X^{2}(s) d s-\phi^{-1}\right)^{2 \gamma} \mid \phi\right) \lesssim T^{-\gamma}\left(\phi^{-3 \gamma}+\phi^{-4 \gamma}\right), \quad \gamma \geq 1
$$

For $V_{T}=\int_{0}^{T} X^{2}(s) d s$ and $p \geq 1$,

$$
\mathbb{P}\left(\frac{V_{T}}{T}<\frac{k}{\sqrt{T}} \mid \phi\right) \lesssim T^{-p}\left(\phi^{2 p}+\phi^{-2 p}+\phi^{-p}\right) .
$$

(Proof in Appendix).

\section{Estimation of the distribution of the RANDOM EFFECT From $N$ i.i.d. TRAJECTORIES.}

The previous sections show that the random effect $\phi$ can be estimated from one trajectory only as $T$ tends to infinity. To estimate parameters in the distribution of $\phi$ requires additional information. In this section, we consider $N$ i.i.d. sample paths $\left(X_{j}(t)\right), j=1, \ldots, N$ satisfying

$$
d X_{j}(t)=b\left(X_{j}(t), \phi_{j}\right) d t+\sigma\left(X_{j}(t)\right) d W_{j}(t), \quad X_{j}(0)=\eta_{j}, j=1, \ldots, N,
$$

where $\left(W_{1}, \ldots, W_{N}\right)$ are $N$ independent Wiener processes, $\left(\phi_{1}, \eta_{1}\right) \ldots,\left(\phi_{N}, \eta_{N}\right)$ are $N$ i.i.d. random variables taking values in $\mathbb{R}^{d} \times \mathbb{R},\left(\left(\phi_{1}, \eta_{1}\right) \ldots,\left(\phi_{N}, \eta_{N}\right)\right)$ and $\left(W_{1}, \ldots, W_{N}\right)$ are independent. We assume that $\left(X_{j}(t), t \leq T\right), j=1, \ldots, N$ are observed. We denote by $\phi_{j}=\left(\phi_{k, j}, k=1, \ldots, d\right)$ the components of $\phi_{j}$.

We assume that $[\mathbf{H 1}]-[\mathbf{H 3}]$ hold, i.e. $\left(\phi_{j}, \eta_{j}\right)$ has distribution $\nu(d \varphi) \otimes \pi_{\varphi}(x) d x$ for some unknown distribution $\nu$. To build estimators of the moments of $\nu$, we rely on the double asymptotic framework $N \rightarrow+\infty$ and $T \rightarrow+\infty$. 
ESTIMATION FOR STOCHASTIC DIFFERENTIAL EQUATIONS WITH MIXED EFFECTS. 11

4.1. Moments method. We first use the moments estimators described in Section 3. Let $g \in L^{1}(\Phi)$ (see $(2.6)$ ) be such that $\mathbb{E}\left|\pi_{\phi}(g)\right|<+\infty$, and set

$$
m_{g}=\mathbb{E}\left(\pi_{\phi}(g)\right)=\int_{(\ell, r) \times \Phi} \pi_{\varphi}(x) g(x) d \nu(\varphi) d x, \quad \widetilde{m_{g}}=\frac{1}{N} \sum_{j=1}^{N} \frac{1}{T} \int_{0}^{T} g\left(X_{j}(s)\right) d s .
$$

In general, $\pi_{\phi_{j}}(g)$ is a random variable. However, if $\phi_{j}$ contains deterministic components (fixed effects), then, $\pi_{\phi_{j}} g$ can be deterministic $\left(\pi_{\phi_{j}} g=\mathbb{E}\left(\pi_{\phi}(g)\right)=m_{g}\right)$. The behaviour of $\widetilde{m_{g}}$ differs according to these two cases.

Theorem 4.1. Assume that $\mathbb{E}\left(V_{\phi}(g)+\rho(g, \phi)\right)<+\infty$ (see (2.10) and (3.5)).

- If $\pi_{\phi_{j}}(g)$ is random and $\mathbb{E}\left(\pi_{\phi}^{2}(g)\right)<+\infty$, then $\sqrt{N}\left(\widetilde{m_{g}}-m_{g}\right)$ converges in distribution to $\mathcal{N}\left(0, \operatorname{Var}\left(\pi_{\phi}(g)\right)\right)$, where $\operatorname{Var}\left(\pi_{\phi}(g)\right)=\mathbb{E}\left(\pi_{\phi}^{2}(g)\right)-m_{g}^{2}$.

- Let $\pi_{\phi_{j}}(g)=m_{g}=\mathbb{E}\left(\pi_{\phi}(g)\right)$ be deterministic. Assume that condition $\mathbf{K}(1+\varepsilon)$ holds with $\varepsilon>0$ and that

$$
\mathbb{E} K_{1+\varepsilon}(\phi, g)<+\infty .
$$

Then $\sqrt{N T}\left(\widetilde{m_{g}}-m_{g}\right)$ converges in distribution to $\mathcal{N}\left(0, \mathbb{E}\left(V_{\phi}(g)\right)\right)$.

We can specialize this result for the case of Model 2 (see 2.1).

Examples 4.1.

Model 2 (continued). For $j=1, \ldots, N$, let $X_{j}$ satisfy Model 2 with $\mathbb{E} \phi_{1, j}^{2}<+\infty, \mathbb{E} \phi_{2, j}^{-3}<$ $+\infty$. Define $m=\mathbb{E} \phi_{1, j}, v=\mathbb{E}\left(\left(\phi_{2, j}\right)^{-1}\right)$ and

$$
\begin{gathered}
\tilde{m}=\frac{1}{N} \sum_{j=1}^{N} \frac{1}{T} \int_{0}^{T} X_{j}(s) d s, \quad \tilde{v}=\frac{1}{N} \sum_{j=1}^{N} \tilde{\tau}_{j} \quad \text { with } \\
\tilde{\tau}_{j}=2\left(\frac{1}{T} \int_{0}^{T} X_{j}^{2}(s) d s-\left(\frac{1}{T} \int_{0}^{T} X_{j}(s) d s\right)^{2}\right) .
\end{gathered}
$$

- If $\phi_{1, j}$ is random, $\sqrt{N}(\tilde{m}-m)$ converges in distribution as both $T, N$ tend to infinity, to $\mathcal{N}\left(0, \operatorname{Var}\left(\phi_{1, j}\right)\right)$.

- If $\phi_{1, j}=m$ is deterministic, $\sqrt{N T}(\tilde{m}-m)$ converges in distribution to $\mathcal{N}\left(0, \mathbb{E}\left(\frac{1}{\phi_{2, j}^{2}}\right)\right)$.

- If $\phi_{2, j}$ is random and $\sqrt{N} / T$ tends to 0 as both $N, T=T(N)$ tend to infinity, then $\sqrt{N}(\tilde{v}-v)$ converges in distribution to $\mathcal{N}\left(0, \operatorname{Var}\left(\tau_{j}\right)\right)$ where $\tau_{j}=\left(\phi_{2, j}\right)^{-1}$.

- If $\phi_{2, j}=v^{-1}$ is deterministic and $N / T$ tends to 0 as both $N, T=T(N)$ tend to infinity, then $\sqrt{N T}(\tilde{v}-v)$ converges in distribution to $\mathcal{N}\left(0,2 v^{3}+12 v^{2}\right)$.

The additional constraint linking $N$ and $T=T(N)$ comes from the fact that $\tilde{\tau}_{j}$ is a conditionally biased estimator of $\tau_{j}=\left(\phi_{2, j}\right)^{-1}$.

(Proof in Appendix).

4.2. Maximum likelihood approach in the case of Model 1 (see 2.1). Suppose that a parametric model rules the $\phi_{j}$ 's, i.e. $\nu(d \varphi)=f(\theta, \varphi) d \alpha(\varphi)$ where $\alpha$ is a dominating measure on $\Phi$ and $\theta \in \Theta$ is unknown. Consider the log-likelihood associated with $\left(\phi_{j}, j=1, \ldots, N\right)$, $\ell_{N}(\theta)=\sum_{j=1}^{N} \log f\left(\theta, \phi_{j}\right)$. As the random variables $\phi_{j}$ 's are not directly observed, a natural strategy consists in plugging in $\ell_{N}(\theta)$ estimators of the $\phi_{j}$ 's based on the results of Section 3. An analogous method is applied in Delattre et al. (2014) for a different mixed effect model and different observations. Such an approach has to rely on a precise model for the diffusion process 
and the parametric model for $\nu$. We choose to develop the method for the Ornstein-Uhlenbeck process with one multiplicative random effect

$$
d X_{j}(t)=-\phi_{j} X_{j}(t) d t+d W_{j}(t), \quad X_{j}(0)=\eta_{j}, j=1, \ldots, N
$$

where $\phi_{j}$ belongs to $\Phi=(0, \infty)$. For the parametric model on $\nu$, we assume that $\nu$ is a Gamma distribution with parameter $\theta=(a, \lambda)$, i.e.

$$
f(\theta, \varphi)=\frac{\lambda^{a}}{\Gamma(a)} \varphi^{a-1} e^{-\lambda \varphi} 1_{\varphi>0} .
$$

Consider the loglikelihood associated with the observation of $\left(\phi_{j}, j=1, \ldots, N\right)$

$$
\ell_{N}(\theta)=N a \log \lambda-N \log \Gamma(a)+(a-1) \sum_{j=1}^{N} \log \phi_{j}-\lambda \sum_{j=1}^{N} \phi_{j}
$$

and define $\theta_{N}=\operatorname{Argmax}_{\theta} \ell_{N}(\theta)$ the maximum likelihood estimator based on the direct observation of $\left(\phi_{i}, i=1, \ldots, N\right)$. The asymptotic behaviour of $\theta_{N}$ is well known. As $N$ tends to infinity, $\theta_{N}$ is consistent and $\sqrt{N}\left(\theta_{N}-\theta\right)$ converges in distribution to the Gaussian distribution $\mathcal{N}_{2}\left(0, I^{-1}(\theta)\right)$ where

$$
I(\theta)=\left(\begin{array}{cc}
\psi^{\prime}(a) & -\lambda^{-1} \\
-\lambda^{-1} & a \lambda^{-2}
\end{array}\right)
$$

and

$$
\psi(a)=\frac{\Gamma^{\prime}(a)}{\Gamma(a)}=-\gamma+\int_{0}^{1} \frac{1-t^{a-1}}{1-t} d t
$$

is the di-Gamma function where $\gamma=-\Gamma^{\prime}(1)$ is the Euler constant. Using that $\psi^{\prime}(a)=-\int_{0}^{1} t^{a-1}(\log t /(1-t)) d t$, yields $a \psi^{\prime}(a)-1 \neq 0$ so that $I(\theta)$ is invertible.

For the plug-in, we must define estimators of both $\phi_{j}$ and $\log \phi_{j}$ with appropriate moments properties. In this model, we can use two different estimators, the moment type estimators given by

$$
\left(\frac{2}{T} \int_{0}^{T} X_{j}^{2}(s) d s\right)^{-1}
$$

or the estimator based on a maximum likelihood approach

$$
\hat{\phi}_{j}=-\frac{\int_{0}^{T} X_{j}(s) d X_{j}(s)}{\int_{0}^{T} X_{j}^{2}(s) d s} .
$$

For both estimators, getting explicit bounds for their moments is not straightforward. Introducing some appropriate truncations is a good tool to obtain these bounds. In Section 3.2, we have applied the truncation method to $\hat{\phi}_{j}$ ( see Corollary 3.3 ). So we use it for the plug-in device. Let us set:

$$
\hat{\phi}_{i}^{(k)}=\hat{\phi}_{i} 1_{\left(\frac{V_{i, T}}{T}>\frac{k}{\sqrt{T}}\right)} \quad \text { with } \quad V_{i, T}=\int_{0}^{T} X_{i}^{2}(s) d s .
$$

This estimator may be negative and null. Therefore we define a specific estimator for $L_{i}=\log \phi_{i}$ and set, for $k$ a constant:

$$
\widehat{L}_{i}^{(k)}=\left(\log \hat{\phi}_{i}\right) 1_{\left(\hat{\phi}_{i} \geq \frac{k}{\sqrt{T}}, \frac{V_{i, T}}{T}>\frac{k}{\sqrt{T}}\right)}=\left(\log \left(\hat{\phi}_{i}^{(k)}\right)\right) 1_{\left(\hat{\phi}_{i} \geq \frac{k}{\sqrt{T}}, \frac{V_{i, T}}{T}>\frac{k}{\sqrt{T}}\right)} .
$$


Now, set

$$
V_{N}(\theta)=N a \log \lambda-N \log \Gamma(a)-\lambda \sum_{i=1}^{N} \hat{\phi}_{i}^{(k)}+(a-1) \sum_{i=1}^{N} \widehat{L}_{i}^{(k)}
$$

and define the estimator of $\theta$ as any solution of:

$$
\tilde{\theta}_{N}=\operatorname{Argmax}_{\theta} V_{N}(\theta) \text {. }
$$

Proposition 4.1. Assume that $a>8$ and that both $N, T$ tend to infinity. Then, $\tilde{\theta}_{N}$ is consistent. If moreover, $N / T$ tends to 0 , then $\sqrt{N}\left(\tilde{\theta}_{N}-\theta_{N}\right)=o_{P}(1)$ where $\theta_{N}$ is the maximum likelihood estimator based on the observation of $\left(\phi_{1}, \ldots, \phi_{N}\right)$.

\section{Concluding REMARKS}

In this paper, we investigate estimation problems linked with the observation of processes defined by an SDE with mixed effects from a continuous observation on a time interval $[0, T]$. Generally, authors are interested in estimating both the fixed effects and unknown parameters in the distribution of the random effects when $N$ i.i.d. processes $\left(\left(X_{j}(t), t \leq T\right), j=1, \ldots, N\right)$ are observed and $N$ tends to infinity. Two cases are investigated: Either $T$ is fixed or $T$ tends to infinity (see references in the introduction). In the latter case, constraints linking $N$ and $T=T(N)$ are required.

In the case $T$ tends to infinity, the interest of our contribution is to consider separately the inference based on one sample path and the inference based on $N$ i.i.d. sample paths. This leads to study the long term behaviour of a one-dimensional SDE with mixed effects. Then, we apply these results to derive estimators of the random variable $\phi$ itself based on functionals of the continuous sample path $(X(t), t \leq T)$. Here, two kinds of estimators of $\phi$ are studied and proved to be consistent and asymptotically mixed normal as $T$ goes to infinity with rate $\sqrt{T}$. The results are applied to various classical examples.

To estimate the common distribution of the $\phi_{j}$ 's when $N$ i.i.d. processes $\left(\left(X_{j}(t), t \leq T\right), j=\right.$ $1, \ldots, N)$ are observed, we proceed as follows. From each sample path $\left(X_{j}(t), t \leq T\right)$ we build an estimator of $\phi_{j}$, say $\tilde{\phi}_{j}$ and replace $\phi_{j}$ by $\tilde{\phi}_{j}$ to obtain estimators of parameters in the distribution of $\phi_{j}$. The method relies on a double asymptotic framework where both $N$ and $T$ go to infinity.

Our approach applies to general diffusion models and to general distributions for the random effects up to some moments constraints. The results obtained in Proposition 3.5 have been extended to the case of a multivariate random effect in the drift in Dion and Genon-Catalot (2015). It is then used to build nonparametric estimators of the joint density of the random effects. The truncated technique introduced for maximum likelihood estimators is quite general and has many potential applications.

\section{Appendix: proofs}

Proof of Proposition 3.1

The process $X^{\varphi}$ defined in (2.14) satisfies the ergodic theorem for all $\varphi \in \Phi$ :

$$
\mathbb{P}\left(\frac{1}{T} \int_{0}^{T} g\left(X^{\varphi}(s)\right) d s \rightarrow_{T \rightarrow+\infty} \pi_{\varphi}(g)\right)=1 .
$$

By Proposition 2.1,

$$
\mathbb{P}\left(\frac{1}{T} \int_{0}^{T} g(X(s)) d s \rightarrow \pi_{\phi}(g) \mid \phi=\varphi\right)=\mathbb{P}\left(\frac{1}{T} \int_{0}^{T} g\left(X^{\varphi}(s)\right) d s \rightarrow \pi_{\varphi}(g)\right) .
$$


Thus, $\mathbb{P}\left(T^{-1} \int_{0}^{T} g(X(s)) d s \rightarrow \pi_{\phi}(g)\right)=1$.

Proof of Proposition 3.2

We consider for fixed $\varphi$ the process $X^{\varphi}$ given by $(2.14)$ and $F_{\varphi, g}$ given by (2.9). By the Ito formula, we have:

$$
F_{\varphi, g}\left(X^{\varphi}(T)\right)=F_{\varphi, g}\left(X^{\varphi}(0)\right)+\int_{0}^{T} \mathcal{L}_{\varphi} F_{\varphi, g}\left(X^{\varphi}(s)\right) d s+\int_{0}^{T} F_{\varphi, g}^{\prime}\left(X^{\varphi}(s)\right) \sigma\left(X^{\varphi}(s)\right) d W(s) .
$$

Therefore,

$$
-\frac{1}{\sqrt{T}} \int_{0}^{T} \mathcal{L}_{\varphi} F_{\varphi, g}\left(X^{\varphi}(s)\right) d s=\frac{1}{\sqrt{T}} \int_{0}^{T} F_{\varphi, g}^{\prime}\left(X^{\varphi}(s)\right) \sigma\left(X^{\varphi}(s)\right) d W(s)+R_{T}^{\varphi},
$$

with

$$
R_{T}^{\varphi}=T^{-1 / 2}\left(F_{\varphi, g}\left(X^{\varphi}(0)\right)-F_{\varphi, g}\left(X^{\varphi}(T)\right) .\right.
$$

The ergodic properties of $X^{\varphi}$ imply that $X^{\varphi}(T)$ converges in distribution to the stationary distribution $\pi_{\varphi}(x) d x$ as $T$ tends to infinity. Hence, $R_{T}^{\varphi}$ tends to 0 in probability. We can conclude that,

$$
-\frac{1}{\sqrt{T}} \int_{0}^{T} \mathcal{L}_{\varphi} F_{\varphi, g}\left(X^{\varphi, g}(s)\right) d s \rightarrow \mathcal{N}\left(0, V_{\varphi}(g)\right) .
$$

Setting $g_{\varphi}=g-\pi_{\varphi} g$, we have

$$
\frac{1}{\sqrt{T}} \int_{0}^{T} g_{\phi}(X(s)) d s=\sqrt{T}\left(\widetilde{\pi_{\phi}(g)}-\pi_{\phi}(g)\right)
$$

By conditioning, we obtain, for $u \in \mathbb{R}, v \in \mathbb{R}^{d}$,

$$
\mathbb{E}\left(\exp \left(i u \frac{1}{\sqrt{T}} \int_{0}^{T} g_{\phi}(X(s)) d s+i v . \phi\right)\right) \rightarrow \mathbb{E}\left(\exp \left(-\frac{u^{2}}{2} V_{\phi}(g)+i v . \phi\right)\right),
$$

which gives the convergence in distribution result.

The multidimensional result is obtained by applying the above result to $\sum_{i=1}^{k} u_{j} g_{j}=\underline{u}^{\prime} \underline{g}$ with the Cramer-Wold device.

Proof of Proposition 3.3

We use the same notation $X^{\varphi}$ for the stationary and ergodic process with fixed $\varphi \in \Phi$ :

$$
\left.d X^{\varphi}(t)=b\left(X^{\varphi}(t)\right), \varphi\right) d t+\sigma\left(X^{\varphi}(t)\right) d W(t), \quad X^{\varphi}(0) \sim \pi_{\varphi}
$$

with $X^{\varphi}(0)$ independent of $W$. The process $X^{\varphi}$ has marginal distribution $\pi_{\varphi}$. Recall the notation $g_{\varphi}=g-\pi_{\varphi} g$. Using (6.1)-(6.2) and the Hölder and the Burkholder-Davis-Gundy inequalities yields:

$$
\mathbb{E}\left(\int_{0}^{T} g_{\varphi}\left(X^{\varphi}(s)\right) d s\right)^{2 \gamma} \leq c_{\gamma}\left(T^{\gamma} \pi_{\varphi}\left(\left(F_{\varphi, g}^{\prime} \sigma(.)\right)^{2 \gamma}+\pi_{\varphi}\left(\left(F_{\varphi, g}\right)^{2 \gamma}\right)\right)\right.
$$

Now,

$$
\mathbb{E}\left(\left(\int_{0}^{T} g_{\phi}(X(s)) d s\right)^{2 \gamma} \mid \phi=\varphi\right)=\mathbb{E}\left(\int_{0}^{T} g_{\varphi}\left(X^{\varphi}(s)\right) d s\right)^{2 \gamma} .
$$


ESTIMATION FOR STOCHASTIC DIFFERENTIAL EQUATIONS WITH MIXED EFFECTS. 15

Hence, the result for all $\gamma \geq 1$ using (6.3) provided that $T \geq 1$.

For $\gamma=1$,

$$
\mathbb{E}\left(\frac{1}{\sqrt{T}} \int_{0}^{T} g_{\varphi}\left(X^{\varphi}(s)\right) d s\right)^{2}=\pi_{\varphi}\left(\left(F_{\varphi, g}^{\prime} \sigma(.)\right)^{2}+Z_{T}(\varphi)=V_{\varphi}(g)+Z_{T}(\varphi)\right.
$$

with $($ see $(6.2))$

$$
Z_{T}(\varphi)=\mathbb{E}\left(R_{T}^{\varphi}\right)^{2}+2 \mathbb{E}\left(R_{T}^{\varphi} \frac{1}{\sqrt{T}} \int_{0}^{T} F_{\varphi, g}^{\prime}\left(X^{\varphi}(s)\right) \sigma\left(X^{\varphi}(s)\right) d W(s)\right) .
$$

We have:

$$
\mathbb{E}\left(R_{T}^{\varphi}\right)^{2} \leq \frac{2}{T} \mathbb{E}\left(F_{\varphi, g}\left(X^{\varphi}(0)\right)\right)^{2}=\frac{2}{T} \pi_{\varphi}\left[\left(F_{\varphi, g}\right)^{2}\right]
$$

And

$$
\begin{aligned}
\left|\mathbb{E}\left(R_{T}^{\varphi} \frac{1}{\sqrt{T}} \int_{0}^{T} F_{\varphi, g}^{\prime}\left(X^{\varphi}(s)\right) \sigma\left(X^{\varphi}(s)\right) d W(s)\right)\right| & \leq\left(\mathbb{E}\left(R_{T}^{\varphi}\right)^{2} \mathbb{E}\left(\frac{1}{T} \int_{0}^{T}\left(F_{\varphi, g}^{\prime}\left(X^{\varphi}(s)\right) \sigma\left(X^{\varphi}(s)\right)\right)^{2} d s\right)\right)^{1 / 2} \\
& \leq\left(\frac{2}{T} \pi_{\varphi}\left[\left(F_{\varphi, g}\right)^{2}\right] V_{\varphi}(g)\right)^{1 / 2}
\end{aligned}
$$

For considering the two functions $g, h$, we just write $g h=\left((g+h)^{2}-(g-h)^{2}\right) / 4$ and apply the previous result.

Proofs of the results in Examples 3.1.

We first treat the Ornstein-Uhlenbeck process with two random effects (Example 2). Consider $g(x)=x, h(x)=x^{2}$. Then, $\pi_{\phi}(g)=\phi_{1}, \pi_{\phi}(h):=\Psi=\phi_{1}^{2}+1 /\left(2 \phi_{2}\right)$,

$$
\widetilde{\pi_{\phi}(g)}=\widetilde{\phi}_{1}=\frac{1}{T} \int_{0}^{T} X(s) d s, \quad \widetilde{\pi_{\phi}(h)}=\widetilde{\Psi}=\frac{1}{T} \int_{0}^{T} X^{2}(s) d s
$$

and $\left(\widetilde{\phi}_{1}, \widetilde{\Psi}\right)$ converges a.s. to $\left(\phi_{1}, \Psi\right)$. For $(2.8)-(2.10)$, we check that

$$
\begin{aligned}
& F_{\varphi, g}(x)=\left(x-\varphi_{1}\right) / \varphi_{2}, \quad F_{\varphi, h}(x)=\frac{\left(x-\varphi_{1}\right)^{2}}{2 \varphi_{2}}+2 \frac{\varphi_{1}}{\varphi_{2}}\left(x-\varphi_{1}\right), \\
& V_{\varphi}(g, g)=\frac{1}{\varphi_{2}^{2}}, \quad V_{\varphi}(h, h)=\frac{1}{2 \varphi_{2}^{3}}+4\left(\frac{\varphi_{1}}{\varphi_{2}}\right)^{2}, \quad V_{\varphi}(g, h)=2 \frac{\varphi_{1}}{\varphi_{2}^{2}} .
\end{aligned}
$$

The matrix $V(\varphi)=V_{\varphi}\left((g h)^{\prime}\right)$ satisfies $\operatorname{det} V(\varphi)=1 /\left(2 \varphi_{2}^{5}\right)>0$. Consequently,

$$
\sqrt{T}\left(\widetilde{\phi}_{1}-\phi_{1}, \widetilde{\Psi}-\Psi\right)^{\prime} \rightarrow_{\mathcal{D}} \mathcal{M N}_{2}(0, V(\phi)) .
$$

Using that $\widetilde{\phi}_{2}=\left(2\left(\widetilde{\Psi}-\left(\widetilde{\phi}_{1}\right)^{2}\right)\right)^{-1}$ and the $\delta$-method, we deduce after some computations that $\sqrt{T}\left(\widetilde{\phi}_{1}-\phi_{1}, \widetilde{\phi}_{2}-\phi_{2}\right)^{\prime} \rightarrow_{\mathcal{D}} \mathcal{M N}_{2}(0, J(\phi))$.

In this model, the conditional distribution of $\widetilde{\phi}_{1}$ is explicit and conditionally Gaussian with mean $\phi_{1}$. To compute the conditional variance, we use the solution of the SDE:

$$
X(t)=\phi_{1}+e^{-\phi_{2} t}\left(\eta-\phi_{1}\right)+e^{-\phi_{2} t} \int_{0}^{t} e^{\phi_{2} s} d W(s) .
$$

As $(X(t))$ is stationary, $\mathbb{E}(X(t) X(t+h) \mid \phi)=\mathbb{E}(X(0) X(h) \mid \phi)=\phi_{1}^{2}+e^{-\phi_{2} h}\left(2 \phi_{2}\right)^{-1}$. Therefore,

$$
\left.\mathbb{E}\left(\left(\widetilde{\phi}_{1}\right)^{2}\right) \mid \phi\right)=2 T^{-2} \int_{0<s<s^{\prime}<T} \phi_{1}^{2}+\frac{e^{-\phi_{2}\left(s^{\prime}-s\right)}}{2 \phi_{2}} d s d s^{\prime}=\phi_{1}^{2}+\Sigma_{T}\left(\phi_{2}\right),
$$


where

$$
\Sigma_{T}\left(\phi_{2}\right)=\frac{1}{T \phi_{2}^{2}}-\frac{1-e^{-\phi_{2} T}}{T^{2} \phi_{2}^{3}} .
$$

Conditionally on $\phi, \widetilde{\phi}_{1}$ has distribution $\mathcal{N}\left(\phi_{1}, \Sigma_{T}\left(\phi_{2}\right)\right)$. In particular,

$$
\begin{gathered}
\left.\mathbb{E}\left(\left(\widetilde{\phi}_{1}-\phi_{1}\right)\right)^{2 \gamma} \mid \phi\right)=C(2 \gamma) \Sigma_{T}^{\gamma}\left(\phi_{2}\right) \quad \text { and } \\
\mathbb{E}\left(\sqrt{T}\left(\left(\widetilde{\phi}_{1}-\phi_{1}\right)\right)^{2} \mid \phi\right)=\frac{1}{\phi_{2}^{2}}+Z_{T}, \quad\left|Z_{T}\right| \leq \frac{1}{T \phi_{2}^{3}} .
\end{gathered}
$$

Note that $\rho(g, \phi)=\phi_{2}^{-3}+2 \phi_{2}^{-5 / 2}$. Thus, the bound for $Z_{T}=Z_{T}(g, \phi)$ given in Proposition 3.3 is really improved by exact computations. We can also check condition $(\mathbf{K} \gamma)$ for $g$ :

$$
K_{\gamma}(\varphi, g)=\pi_{\varphi}\left(F_{\varphi, g}^{2 \gamma}\right)+\left(\pi_{\varphi}\left(\left(F_{\varphi, g}^{\prime} \sigma\right)^{2 \gamma}\right)=\frac{C(2 \gamma)}{2^{\gamma} \varphi_{2}^{3 \gamma}}+\frac{1}{\varphi_{2}^{2 \gamma}}\right.
$$

where $C(2 \gamma)=\mathbb{E} X^{2 \gamma}$ for $X$ a standard Gaussian variable. Therefore, by (3.6),

$$
\left.\mathbb{E}\left(\widetilde{\phi}_{1}-\phi_{1}\right)^{2 \gamma} \mid \phi\right) \lesssim T^{-\gamma}\left(\phi_{2}^{-3 \gamma}+\phi_{2}^{-2 \gamma}\right) .
$$

Comparing with the exact result (6.8), we see that this bound is accurate.

Next, we study the estimation of $\tau=\phi_{2}^{-1}$ by $\widetilde{\tau}=\widetilde{\phi}_{2}^{-1}=2\left(\widetilde{\Psi}-\left(\widetilde{\phi}_{1}\right)^{2}\right)$. Note that, using the Cauchy-Schwarz inequality and that $t \rightarrow X(t)$ is not constant, $\left(\widetilde{\phi}_{1}\right)^{2}<\widetilde{\Psi}$, so that $\widetilde{\tau}>0$. We have

$$
\mathbb{E}(\widetilde{\tau} \mid \phi)=\tau-2 \Sigma_{T}\left(\phi_{2}\right) .
$$

We now use the decomposition

$$
\widetilde{\tau}-\tau=2\left[(\widetilde{\Psi}-\Psi)-\left(\widetilde{\phi}_{1}-\phi_{1}\right)^{2}-2 \phi_{1}\left(\widetilde{\phi}_{1}-\phi_{1}\right)\right] .
$$

By (3.6)-(3.7) with $g$ replaced by $h(x)=x^{2}$ (see also (6.6)), we obtain

$$
\begin{gathered}
\mathbb{E}\left((\widetilde{\Psi}-\Psi)^{2 \gamma} \mid \phi\right) \lesssim T^{-\gamma}\left(\phi_{2}^{-4 \gamma}+\phi_{2}^{-3 \gamma}+\phi_{1}^{2 \gamma} \phi_{2}^{-3 \gamma}+\phi_{1}^{2 \gamma} \phi_{2}^{-2 \gamma}\right), \\
\left.\mathbb{E}(\sqrt{T}(\widetilde{\Psi}-\Psi))^{2} \mid \phi\right)=\frac{1}{2 \phi_{2}^{3}}+4\left(\frac{\phi_{1}}{\phi_{2}}\right)^{2}+Z_{T}(h, \phi) .
\end{gathered}
$$

where $\left|Z_{T}(h, \phi)\right| \leq(1 / \sqrt{T}) \rho(h, \phi)$ and $\rho(h, \phi)$ is given by (3.5). Using (6.5)-(6.6), we obtain

$$
\pi_{\phi} F_{\phi, h}^{2}=\frac{3}{16 \phi_{2}^{4}}+2 \frac{\phi_{1}^{2}}{\phi_{2}^{3}} \text { and } \rho(h, \phi) \lesssim\left(\frac{1}{\phi_{2}^{4}}+\frac{1}{\phi_{2}^{7 / 2}}+\frac{\phi_{1}^{2}}{\phi_{2}^{3}}+\frac{\left|\phi_{1}\right|}{\phi_{2}^{3}}+\frac{\phi_{1}^{2}}{\phi_{2}^{5 / 2}}\right) \text {. }
$$

By (6.13), we have

$$
\left.(\widetilde{\tau}-\tau)^{2 \gamma} \lesssim(\widetilde{\Psi}-\Psi)^{2 \gamma}+\phi_{1}^{2 \gamma}\left(\widetilde{\phi}_{1}-\phi_{1}\right)^{2 \gamma}+\left(\widetilde{\phi}_{1}-\phi_{1}\right)^{4 \gamma}\right) .
$$

Therefore, combining (6.11)-(6.14) yields:

$$
\mathbb{E}\left((\widetilde{\tau}-\tau)^{2 \gamma} \mid \phi\right) \lesssim T^{-\gamma}\left(\phi_{2}^{-2 \gamma}+\phi_{2}^{-4 \gamma}+\phi_{2}^{-3 \gamma}+\phi_{1}^{2 \gamma} \phi_{2}^{-3 \gamma}+\phi_{1}^{2 \gamma} \phi_{2}^{-2 \gamma}+\phi_{2}^{-6 \gamma}\right) .
$$

Moreover,

$$
\mathbb{E}\left((\widetilde{\tau}-\tau)^{2} \mid \phi\right)=4 \sum_{i=1}^{6} A_{i} \quad \text { where }
$$


ESTIMATION FOR STOCHASTIC DIFFERENTIAL EQUATIONS WITH MIXED EFFECTS. 17

$A_{1}=\mathbb{E}\left((\widetilde{\Psi}-\Psi)^{2} \mid \phi\right)$ is given in $(6.15), A_{2}=4 \mathbb{E}\left(\left(\phi_{1}^{2}\left(\widetilde{\phi}_{1}-\phi_{1}\right)^{2} \mid \phi\right)=4 \phi_{1}^{2} \Sigma_{T}\left(\phi_{2}\right)\right.$,

$$
A_{3}=\mathbb{E}\left(\left(\widetilde{\phi}_{1}-\phi_{1}\right)^{4} \mid \phi\right)=3\left(\Sigma_{T}\left(\phi_{2}\right)\right)^{2}, \quad A_{4}=4 \mathbb{E}\left(\phi_{1}\left(\widetilde{\phi}_{1}-\phi_{1}\right)^{3} \mid \phi\right)=0 .
$$

Using (3.8) and $V_{\phi}(h, g)=2 \phi_{1} / \phi_{2}^{2}$ yields

$$
\left.A_{5}=-4 \mathbb{E}\left(\phi_{1}(\widetilde{\Psi}-\Psi)\left(\widetilde{\phi}_{1}-\phi_{1}\right) \mid \phi\right)=-\frac{4 \phi_{1}}{T}\left(\frac{2 \phi_{1}}{\phi_{2}^{2}}\right)+Z_{T}(h, g, \phi)\right) .
$$

Finally, by the Cauchy-Schwarz inequality,

$$
\left|A_{6}\right|=\mid-2 \mathbb{E}\left((\widetilde{\Psi}-\Psi)\left(\left(\widetilde{\phi}_{1}-\phi_{1}\right)^{2} \mid \phi\right) \mid \leq\left(\mathbb{E}\left((\widetilde{\Psi}-\Psi)^{2} \mid \phi\right) \mathbb{E}\left(\left(\widetilde{\phi}_{1}-\phi_{1}\right)^{4} \mid \phi\right)\right)^{1 / 2} .\right.
$$

We obtain

$$
\mathbb{E}\left(\sqrt{T}(\widetilde{\tau}-\tau)^{2} \mid \phi\right)=\frac{2}{\phi_{2}^{3}}+\zeta_{T} \quad \text { with } \quad\left|\zeta_{T}\right| \leq \frac{1}{\sqrt{T}} C(\phi) .
$$

The exact expression of $C(\phi)$ can be derived by some cumbersome computations. If, for $\gamma=$ 1 , the r.h.s. of (6.17) has finite expectation, then $\mathbb{E}(C(\phi))<+\infty$. This yields the result substituting $\phi_{2}^{-1}$ by $\tau$.

Next, we deal with the C.I.R. process with two random effects (Example 3).

We again set $g(x)=x$ and $h(x)=x^{2}$. Then,

$$
\pi_{\phi}(g)=\phi_{1}, \quad \pi_{\phi}(h)=\phi_{1}^{2}+\beta \quad \text { with } \quad \beta=\frac{\phi_{1}}{2 \phi_{2}} .
$$

Some computations lead to

$$
F_{\varphi, g}(x)=\frac{x-\varphi_{1}}{\varphi_{2}}, F_{\varphi, h}(x)=\frac{\left(x-\varphi_{1}\right)^{2}}{2 \varphi_{2}}+\left(x-\varphi_{1}\right)\left(\frac{1}{2 \phi_{2}^{2}}+2 \frac{\phi_{1}}{\phi_{2}}\right) .
$$

We need the moments and the centered moments of a Gamma $G(a, \lambda)$ distribution (recall that $\left.a=2 \varphi_{1} \varphi_{2}, \lambda=2 \varphi_{2}\right)$ : for $k$ integer and $a \geq 1$

$$
\begin{gathered}
\int x^{k} d G(a, \lambda)(x)=\frac{(a+k-1) \ldots(a+1) a}{\lambda^{k}} \leq \lambda^{-k} k ! a^{k}, \\
\int\left(x-\frac{a}{\lambda}\right)^{k} d G(a, \lambda)(x)=\lambda^{-k} \sum_{j=0}^{k}\left(\begin{array}{c}
k \\
j
\end{array}\right)(-a)^{k-j}(a+j-1) \ldots(a+1) a .
\end{gathered}
$$

For $a \geq 1$,

$$
\left|\int\left(x-\frac{a}{\lambda}\right)^{k} d G(a, \lambda)(x)\right| \leq a^{k} \lambda^{-k} \sum_{j=0}^{k} \frac{k !}{(k-j) !}
$$

This leads to:

$$
V_{\varphi}(g, g)=\frac{\varphi_{1}}{\varphi_{2}^{2}}, \quad V_{\varphi}(g, h)=\frac{\varphi_{1}}{\varphi_{2}^{3}}+2 \frac{\varphi_{1}^{2}}{\varphi_{2}^{2}}, \quad V_{\varphi}(h, h)=\frac{5 \varphi_{1}}{4 \varphi_{2}^{4}}+\frac{9 \varphi_{1}^{2}}{2 \varphi_{2}^{3}}+\frac{4 \varphi_{1}^{3}}{\varphi_{2}^{2}} .
$$

The matrix $V:=V_{\varphi}\left((g h)^{\prime}\right)$ satisfies $\operatorname{det} V=\varphi_{1}^{2} /\left(4 \varphi_{2}^{6}\right)+\varphi_{1}^{3} /\left(2 \varphi_{2}^{5}\right)>0$. We apply Propositions 3.1 and 3.2 together with the $\delta$-method to compute $J_{\text {cir }}(\phi)$ and obtain the first part. Now, we look at conditional moments. Using

$$
X(t)=\phi_{1}+e^{-\phi_{2} t}\left(\eta-\phi_{1}\right)+e^{-\phi_{2} t} \int_{0}^{t} e^{\phi_{2} s} \sqrt{X(s)} d W(s)
$$


we find $\mathbb{E}(X(t) X(t+h) \mid \phi)=\mathbb{E}(X(0) X(h) \mid \phi)=\phi_{1}^{2}+\frac{\phi_{1}}{2 \phi_{2}} e^{-\phi_{2} h}$. Therefore,

$$
\left.\mathbb{E}\left(\left(\widetilde{\phi}_{1}\right)^{2}\right) \mid \phi\right)=\phi_{1}^{2}+\phi_{1} \Sigma_{T}\left(\phi_{2}\right),
$$

with $\Sigma_{T}\left(\phi_{2}\right)$ given in (6.7).

Finally, we get, for $\gamma$ integer, $K_{\gamma}(\varphi, g) \lesssim \varphi_{2}^{-2 \gamma}\left(\varphi_{1}^{2 \gamma}+\varphi_{1}^{\gamma}\right)$.

Proof of Proposition 3.5

We have: $\sqrt{T}\left(\hat{\phi}_{T}^{(k)}-\phi\right)=A_{1}+A_{2}$, with

$$
A_{1}=-\sqrt{T} \phi 1_{\left(\frac{V_{T}}{T}<\frac{k}{\sqrt{T}}\right)}, \quad A_{2}=\frac{M_{T} / \sqrt{T}}{V_{T} / T} 1_{\left(\frac{V_{T}}{T} \geq \frac{k}{\sqrt{T}}\right)},
$$

where $M_{T}=\int_{0}^{T}(b(X(s)) / \sigma(X(s))) d W(s)$. Recall that $h=b^{2} / \sigma^{2}$. We first study the term $A_{2}$. Note that:

$$
\mathbb{E}\left[\frac{V_{T}}{T} \mid \phi\right]=\frac{1}{T} \int_{0}^{T} \mathbb{E}[h(X(s)) \mid \phi] d s,
$$

and $\mathbb{E}[h(X(s)) \mid \phi=\varphi]=\mathbb{E}\left[h\left(X^{\varphi}(s)\right)\right]=\pi_{\varphi}(h)$. Thus,

$$
\left.L=\pi_{\phi}(h)=\mathbb{E}\left(\frac{V_{T}}{T} \mid \phi\right)=\mathbb{E} h(X(0)) \mid \phi\right) .
$$

We have: $A_{2}=A_{2}^{\prime}+A_{2}^{\prime \prime}$ with

$$
A_{2}^{\prime}=\frac{M_{T}}{\sqrt{T}}\left(\frac{1}{V_{T} / T}-\frac{1}{L}\right) 1_{\left(\frac{V_{T}}{T} \geq \frac{k}{\sqrt{T}}\right)}, \quad A_{2}^{\prime \prime}=\frac{M_{T}}{L \sqrt{T}} 1_{\left(\frac{V_{T}}{T} \geq \frac{k}{\sqrt{T}}\right)} .
$$

Using that $L$ is $\mathcal{F}_{0}$-measurable, $\left\langle M>_{T}=V_{T}\right.$, the Burkholder-Davis-Gundy and the Hölder inequalities, we get:

$$
\mathbb{E}\left(\left(A_{2}^{\prime \prime}\right)^{2 \gamma} \mid \phi\right) \leq C_{2 \gamma} \mathbb{E}\left(\frac{V_{T}^{\gamma}}{L^{2 \gamma} T^{\gamma}} \mid \phi\right) \leq C_{2 \gamma} \mathbb{E}\left(\frac{1}{L^{2 \gamma} T} \int_{0}^{T} h^{\gamma}(X(s)) d s \mid \phi\right)=C_{2 \gamma} \frac{\pi_{\phi}\left(h^{\gamma}\right)}{L^{2 \gamma}},
$$

where $C_{2 \gamma}$ is the constant of the B-D-G inequality. We need $\pi_{\phi}\left(h^{\gamma}\right)<+\infty$.

For $\gamma=1$, we have $\mathbb{E}\left(\left(A_{2}^{\prime \prime}\right)^{2} \mid \phi\right) \leq L^{-1}$. We look at $A_{2}^{\prime}$ :

$$
\left.\mathbb{E}\left(\left(A_{2}^{\prime}\right)^{2 \gamma} \mid \phi\right) \leq \frac{1}{k^{2 \gamma}}\left(\mathbb{E}\left(\left(\frac{M_{T}}{L \sqrt{T}}\right)^{4 \gamma} \mid \phi\right) \mathbb{E}\left(\left(\sqrt{T}\left(L-V_{T} / T\right)\right)^{4 \gamma} \mid \phi\right)\right)\right)^{1 / 2}
$$

We have:

$$
\mathbb{E}\left(\left(\frac{M_{T}}{L \sqrt{T}}\right)^{4 \gamma} \mid \phi\right) \leq C_{4 \gamma} \mathbb{E}\left(\frac{1}{L^{4 \gamma}}\left(\frac{V_{T}}{T}\right)^{2 \gamma} \mid \phi\right) \leq C_{4 \gamma} \frac{\pi_{\phi}\left(h^{2 \gamma}\right)}{L^{4 \gamma}} .
$$

Therefore, we need $\pi_{\phi}\left(h^{2 \gamma}\right)<+\infty$. Then, we apply Proposition 3.3 and get:

$$
\mathbb{E}\left(\left(\sqrt{T}\left(L-V_{T} / T\right)\right)^{4 \gamma} \mid \phi\right) \lesssim K_{2 \gamma}(\phi, h) .
$$

There remains the term $A_{1}$. We have $A_{1}^{2 \gamma} \leq T^{\gamma} \phi^{2 \gamma} 1_{\left(\frac{V_{T}}{T}<\frac{k}{\sqrt{T}}\right)}$. Therefore, setting $\ell=\pi_{\varphi} h$, we have, for all $p \geq 1$,

$$
\begin{aligned}
\mathbb{P}\left(\frac{V_{T}}{T}<\frac{k}{\sqrt{T}} \mid \phi=\varphi\right) & \leq \mathbb{P}\left(\left|\ell-\frac{V_{T}}{T}\right|>\ell-\frac{k}{\sqrt{T}} \mid \phi=\varphi\right) 1_{\left(\ell>\frac{2 k}{\sqrt{T}}\right)}+1_{\left(\ell \leq \frac{2 k}{\sqrt{T}}\right)} \\
& \leq \mathbb{P}\left(\left|\ell-\frac{V_{T}}{T}\right|>\frac{\ell}{2} \mid \phi=\varphi\right)+1_{\left(\ell^{-1} \geq \frac{\sqrt{T}}{2 k}\right)} \\
& \left.\leq\left(\frac{2}{\ell}\right)^{2 p} \mathbb{E}\left(\ell-\frac{V_{T}}{T}\right)^{2 p} \mid \phi=\varphi\right)+\left(\frac{2 k}{\sqrt{T}}\right)^{2 p} \ell^{-2 p}
\end{aligned}
$$


ESTIMATION FOR STOCHASTIC DIFFERENTIAL EQUATIONS WITH MIXED EFFECTS. 19

Thus, by Proposition 3.3, we get

$$
\begin{aligned}
\mathbb{E}\left(A_{1}^{2 \gamma} \mid \phi\right) & \left.\leq T^{\gamma-p}\left[\phi^{2 \gamma}\left(\frac{2}{L}\right)^{2 p} K_{p}(\phi, h)\right]+T^{\gamma-p}(2 k)^{2 p} \phi^{2 \gamma} L^{-2 p}\right) \\
& \lesssim T^{\gamma-p} \phi^{2 \gamma} L^{-2 p}\left(1+K_{p}(\phi, h)\right)
\end{aligned}
$$

We need $p \geq \gamma$ and that the left-hand side be finite. We take $p=\gamma$.

Proof of the results in Example 3.3

With $h(x)=x^{2}$, in this model, we have $F_{\varphi, h}(x)=(2 \varphi)^{-1}\left(x^{2}-(2 \varphi)^{-1}\right), F_{\varphi, h}^{\prime}(x)=x / \varphi$. This yields for $p \geq 1$ :

$$
V_{\varphi}(h)=\frac{1}{2 \varphi^{3}}, \quad \pi_{\varphi}\left(h^{p}\right)=C(2 p)(2 \varphi)^{-p}, \quad H(p, \varphi, h) \lesssim\left(\varphi^{-3 p}+\varphi^{-4 p}\right) .
$$

We compute $C(\gamma, \phi)$ (Proposition 3.5) and get the result.

Proof of Theorem 4.1 Assume that $\pi_{\phi_{j}}(g)$ is random. We write $\sqrt{N}\left(\widetilde{m_{g}}-m_{g}\right)=T_{1}+T_{2}$ with

$$
\left.T_{1}=\frac{1}{\sqrt{N}} \sum_{j=1}^{N}\left(\frac{1}{T} \int_{0}^{T} g\left(X_{j}(s)\right) d s-\pi_{\phi_{j}}(g)\right), \quad T_{2}=\frac{1}{\sqrt{N}} \sum_{i=1}^{N}\left(\pi_{\phi_{j}}(g)\right)-m_{g}\right) .
$$

The term $T_{2}$ is ruled by the standard central limit theorem and converges in distribution to $\mathcal{N}\left(0, \operatorname{Var}\left(\pi_{\phi}(g)\right)\right)$.

For $T_{1}$, we write:

$$
T_{1}=\sum_{j \leq N} A_{N, j}, \quad A_{N, j}=\frac{1}{\sqrt{N}}\left(\frac{1}{T} \int_{0}^{T} g\left(X_{j}(s)\right) d s-\pi_{\phi_{j}}(g)\right) .
$$

We use the simple Lemma.

Lemma 6.1. Let $\xi_{N}=\sum_{j=1}^{N} \xi_{N, j}$ where conditionally on $\underline{\phi}=\left(\phi_{1}, \ldots, \phi_{N}\right)$, the $\xi_{N, j}$ 's are independent. If $\sum_{j=1}^{N} \mathbb{E}\left(\xi_{N, j} \mid \underline{\phi}\right) \rightarrow \xi$ and $\sum_{j=1}^{N} \mathbb{E}\left(\xi_{N, j}^{2} \mid \underline{\phi}\right) \rightarrow 0$ in probability, then $\xi_{N} \rightarrow \xi$ in probability.

Proof of Lemma 6.1

$$
\xi_{N}-\xi=\sum_{j=1}^{N}\left(\xi_{N, j}-\mathbb{E}\left(\xi_{N, j} \mid \underline{\phi}\right)\right)+\sum_{j=1}^{N} \mathbb{E}\left(\xi_{N, j} \mid \underline{\phi}\right)-\xi:=D_{N}+\sum_{j=1}^{N} \mathbb{E}\left(\xi_{N, j} \mid \underline{\phi}\right)-\xi .
$$

We only need to prove that $D_{N}$ tends to 0 . Using the conditional independence, we have:

$$
\mathbb{E}\left[D_{N}^{2} \mid \underline{\phi}\right]=\sum_{j \leq N}\left(\mathbb{E}\left(\xi_{N, j}^{2} \mid \underline{\phi}\right)-\left[\mathbb{E}\left(\xi_{N, j} \mid \underline{\phi}\right)\right]^{2}\right) \leq \sum_{j \leq N} \mathbb{E}\left(\xi_{N, j}^{2} \mid \underline{\phi}\right) \rightarrow 0 .
$$

Hence, for $h>0, \mathbb{P}\left(\left|D_{N}\right| \geq h \mid \underline{\phi}\right) \leq h^{-2} \mathbb{E}\left[D_{N}^{2} \mid \underline{\phi}\right] \rightarrow 0$. This implies $\mathbb{P}\left(\left|D_{N}\right| \geq h\right) \rightarrow 0 . \square$

Let us apply Lemma 6.1. Conditionally to $\underline{\phi}$, the $A_{N, j}$ 's are independent, centered and by Proposition 3.3,

$$
\sum_{j \leq N} \mathbb{E}\left(A_{N, j}^{2} \mid \underline{\phi}\right)=\frac{1}{T}\left(\frac{1}{N} \sum_{j \leq N}\left(V_{\phi_{j}}(g)+Z_{T}\left(g, \phi_{j}\right)\right)\right)
$$


where $\left|Z_{T}\left(g, \phi_{j}\right)\right| \leq(1 / \sqrt{T}) \rho\left(g, \phi_{j}\right)$. By assumption, $\mathbb{E}\left(V_{\phi_{j}}(g)+\rho\left(g, \phi_{j}\right)\right)<+\infty$. This implies, as both $N$ and $T$ tend to infinity, $\sum_{j \leq N} \mathbb{E}\left(A_{N, j}^{2} \mid \underline{\phi}\right) \rightarrow_{a . s .} 0$. Thus, $T_{1} \rightarrow 0$.

Assume now that $\pi_{\phi_{j}}(g)=m_{g}$ is deterministic. Then,

$$
\sqrt{N T}\left(\widetilde{m_{g}}-m_{g}\right)=\sum_{j \leq N} B_{N, j}, \quad B_{N, j}=\sqrt{T} A_{N, j} .
$$

As above, given $\underline{\phi}$, the $B_{N, j}$ 's are independent, centered and

$$
\sum_{j \leq N} \mathbb{E}\left(B_{N, j}^{2} \mid \underline{\phi}\right)=\left(\frac{1}{N} \sum_{j \leq N}\left(V_{\phi_{j}}(g)+Z_{T}\left(g, \phi_{j}\right)\right)\right) \rightarrow_{a . s .} \mathbb{E} V_{\phi}(g) .
$$

To complete the proof of the convergence in distribution, we check a Lyapounov condition (see e.g. Hall and Heyde (1980)). For $\varepsilon>0$, using Proposition $3.3(\gamma=1+\varepsilon)$,

$$
\sum_{j \leq N} \mathbb{E}\left(B_{N, j}^{2(1+\varepsilon)} \mid \underline{\phi}\right) \leq \frac{c_{1+\varepsilon}}{N^{\varepsilon}} \frac{1}{N} \sum_{j \leq N} K_{1+\varepsilon}\left(\phi_{j}, g\right) \rightarrow_{a . s .} 0 .
$$

Therefore, conditionally on $\underline{\phi}, \sqrt{N T}\left(\widetilde{m_{g}}-m_{g}\right)$ converges in distribution to $\mathcal{N}\left(0, \mathbb{E} V_{\phi}(g)\right)$. Thus, the result.

Proof of the results in Examples 4.1 Let $m=\mathbb{E} \phi_{1, j}$. We apply the result of Theorem 4.1 to the estimation of $m$ with $g(x)=x$. We have $\pi_{\phi_{j}}(g)=\phi_{1, j}$. We use formulae (6.5)-(6.10):

$$
\mathbb{E} V_{\phi_{j}}(g)=\mathbb{E}\left(\frac{1}{\phi_{2, j}^{2}}\right), \quad \mathbb{E} \pi_{\phi_{j}}\left(F_{\phi_{j}, g}\right)^{2}=\mathbb{E}\left(\frac{1}{2 \phi_{2, j}^{3}}\right) .
$$

From (3.5), $\mathbb{E} \rho\left(g, \phi_{j}\right)<+\infty$ holds if $\mathbb{E} V_{\phi_{j}}(g)<+\infty$ and $\mathbb{E} \pi_{\phi_{j}}\left(F_{\phi_{j}, g}^{2}\right)<+\infty$. If $\phi_{1, j}$ is random and $\mathbb{E}\left(\frac{1}{\phi_{2, j}^{3}}\right)<+\infty, \sqrt{N}(\tilde{m}-m)$ converges in distribution as both $T, N$ tend to infinity, to $\mathcal{N}\left(0, \operatorname{Var}\left(\phi_{1, j}\right)\right)$.

If $\phi_{1, j}=m$ is deterministic and $\mathbb{E}\left(\frac{1}{\phi_{2, j}^{3}}\right)<+\infty$ (as $\tilde{m}$ is Gaussian, the condition $\mathbb{E}\left(\frac{1}{\phi_{2, j}^{3(1+\varepsilon)}}\right)<+\infty$ for some $\varepsilon>0$ is not required $), \sqrt{N T}(\tilde{m}-m)$ converges in distribution to $\mathcal{N}\left(0, \mathbb{E}\left(\frac{1}{\phi_{2, j}^{2}}\right)\right)$. This is consistent with relation (6.9) obtained for one trajectory.

Now, we consider the estimation of $v=\mathbb{E}\left(\left(\phi_{2, j}\right)^{-1}\right)$. Assume that

$$
\tau_{j}=\left(\phi_{2, j}\right)^{-1}
$$

is random. We use the decomposition $\sqrt{N}(\tilde{v}-v)=T_{1}+T_{2}$ with

$$
T_{1}=\sum_{j \leq N} A_{N, j}, \quad A_{N, j}=\frac{1}{\sqrt{N}}\left(\tilde{\tau}_{j}-\tau_{j}\right), \quad T_{2}=\frac{1}{\sqrt{N}} \sum_{j=1}^{N}\left(\tau_{j}-v\right) .
$$

The term $T_{2}$ is ruled by the classical central limit theorem. Provided that $\mathbb{E}\left(\tau_{j}^{2}\right)<+\infty, T_{2}$ converges in distribution to $\mathcal{N}\left(0, \operatorname{Var}\left(\tau_{j}\right)\right)$. For the term $T_{1}$, we have:

$$
\mathbb{E}\left(A_{N, j} \mid \underline{\phi}\right)=-\frac{2}{\sqrt{N}} \Sigma_{T}\left(\phi_{2, j}\right)=-2 \frac{\sqrt{N}}{T} \frac{1}{N} \sum_{j \leq N}\left(\frac{1}{\phi_{2, j}^{2}}+\frac{1-e^{-\phi_{2, j} T}}{T \phi_{2, j}^{3}}\right) .
$$

If $\sqrt{N} / T$ tends to 0 and $\mathbb{E}\left(\phi_{2}^{-3}\right)<+\infty, \sum_{j \leq N} \mathbb{E}\left(A_{N, j} \mid \underline{\phi}\right)$ tends a.s. to 0. Moreover,

$$
\sum_{j \leq N} \mathbb{E}\left(A_{N, j}^{2} \mid \underline{\phi}\right) \rightarrow 0 \text {. }
$$


ESTIMATION FOR STOCHASTIC DIFFERENTIAL EQUATIONS WITH MIXED EFFECTS. 21

Therefore, $\sqrt{N}(\tilde{v}-v)$ converges in distribution, as $T, N$ tend to infinity and $\sqrt{N} / T$ tends to 0 , to $\mathcal{N}\left(0, \operatorname{Var}\left(\tau_{j}\right)\right)$.

Assume that $\tau_{j}=v$ is deterministic. Then $\sqrt{N T}(\tilde{v}-v)=\frac{\sqrt{T}}{\sqrt{N}} \sum_{j \leq N}\left(\tilde{\tau}_{j}-v\right)=\sqrt{T} \sum_{j \leq N} A_{N, j}$. If $N / T$ tends to 0 ,

$$
\sqrt{T} \mathbb{E}\left(A_{N, j} \mid \underline{\phi}\right)=-\frac{2 \sqrt{T}}{\sqrt{N}} \sum_{j \leq N} \Sigma_{T}\left(\phi_{2, j}\right)=-2 \frac{\sqrt{N}}{\sqrt{T}} \frac{1}{N} \sum_{j \leq N}\left(\frac{1}{\phi_{2, j}^{2}}-\frac{1-e^{-\phi_{2, j} T}}{T \phi_{2, j}^{3}}\right) \rightarrow 0 .
$$

Moreover,

$$
T \sum_{j \leq N} \mathbb{E}\left(A_{N, j}^{2} \mid \underline{\phi}\right) \rightarrow 2 v^{3}
$$

In this case, $\sqrt{N T}(\tilde{v}-v)$ converges in distribution to a centered Gaussian random variable with variance $2 v^{3}$.

Proof of Proposition 4.1 We have

$$
\begin{gathered}
\frac{1}{N}\left(V_{N}(\theta)-\ell_{N}(\theta)\right)=\frac{a-1}{N} \sum_{i=1}^{N}\left(\widehat{L}_{i}^{(k)}-\log \phi_{i}\right)-\frac{\lambda}{N} \sum_{i=1}^{N}\left(\hat{\phi}_{i}^{(k)}-\phi_{i}\right), \\
\frac{1}{\sqrt{N}}\left(\frac{\partial V_{N}}{\partial a}(\theta)-\frac{\partial \ell_{N}}{\partial a}(\theta)\right)=\frac{1}{\sqrt{N}} \sum_{i=1}^{N}\left(\widehat{L}_{i}^{(k)}-\log \phi_{i}\right) \\
\frac{1}{\sqrt{N}}\left(\frac{\partial V_{N}}{\partial \lambda}(\theta)-\frac{\partial \ell_{N}}{\partial \lambda}(\theta)\right)=\frac{1}{\sqrt{N}} \sum_{i=1}^{N}\left(\hat{\phi}_{i}^{(k)}-\phi_{i}\right)
\end{gathered}
$$

and

where

$$
\frac{\partial^{2} V_{N}}{\partial a^{2}}(\theta)=\frac{\partial^{2} \ell_{N}}{\partial a^{2}}(\theta), \frac{\partial^{2} V_{N}}{\partial a \partial \lambda}(\theta)=\frac{\partial^{2} \ell_{N}}{\partial a \partial \lambda}(\theta), \frac{\partial^{2} V_{N}}{\partial \lambda^{2}}(\theta)=\frac{\partial^{2} \ell_{N}}{\partial \lambda^{2}}(\theta)
$$

$$
-\frac{1}{N} \partial^{2} \ell_{n}(\theta)=I(\theta)
$$

To obtain the announced result, it is enough to prove that, as both $N, T$ tend to infinity,

$$
\frac{1}{N} \sum_{i=1}^{N}\left(\widehat{L}_{i}^{(k)}-\log \phi_{i}\right)=o_{P}(1), \quad \frac{1}{N} \sum_{i=1}^{N}\left(\hat{\phi}_{i}^{(k)}-\phi_{i}\right)=o_{P}(1),
$$

and that, if moreover $N / T$ tends to 0 ,

$$
\frac{1}{\sqrt{N}} \sum_{i=1}^{N}\left(\widehat{L}_{i}^{(k)}-\log \phi_{i}\right)=o_{P}(1), \quad \frac{1}{\sqrt{N}} \sum_{i=1}^{N}\left(\hat{\phi}_{i}^{(k)}-\phi_{i}\right)=o_{P}(1) .
$$

We have, applying Corollary 3.3 with $\gamma=1$ :

$$
\begin{aligned}
\mathbb{E}\left(\sum_{i=1}^{N}\left(\hat{\phi}_{i}^{(k)}-\phi_{i}\right)\right)^{2} & \left.=N \mathbb{E}\left(\hat{\phi}_{1}^{(k)}-\phi_{1}\right)^{2}+N(N-1)\left(\mathbb{E}\left(\hat{\phi}_{1}^{(k)}\right)-\phi_{1}\right)\right)^{2} \\
& \lesssim \frac{N+N(N-1)}{T}\left(1+\mathbb{E}\left(\phi_{1}+\phi_{1}^{4}+\phi_{1}^{-2}+\phi_{1}^{-3}\right)\right.
\end{aligned}
$$


Thus, $\frac{1}{N} \sum_{i=1}^{N}\left(\hat{\phi}_{i}^{(k)}-\phi_{i}\right)=o_{P}(1)$ if both $T, N$ tend to infinity. Under the additional constraint $N / T \rightarrow 0, \frac{1}{\sqrt{N}} \sum_{i=1}^{N}\left(\hat{\phi}_{i}^{(k)}-\phi_{i}\right)=o_{P}(1)$.

To complete the proof, it remains to study $\mathbb{E}\left(\widehat{L}_{1}^{(k)}-\log \phi_{1}\right)^{2}$.

Lemma 6.2. If $a>8, \mathbb{E}\left(\widehat{L}_{1}^{(k)}-\log \phi_{1}\right)^{2} \lesssim T^{-1}$.

Proof of Lemma 6.2. As we deal with only one trajectory, we drop the index 1 in all notations. Set $A=\left(\hat{\phi} \geq k / \sqrt{T}, V_{T} / T \geq k / \sqrt{T}\right)$. We write:

$$
\widehat{L}^{(k)}-\log \phi=(\log \hat{\phi}-\log \phi) 1_{A}-\log \phi 1_{A^{c}}:=T_{1}+T_{2} .
$$

On the set $A, \hat{\phi}=\hat{\phi}^{(k)}$. Applying the Taylor formula yields:

$T_{1}=1_{A}\left(\hat{\phi}^{(k)}-\phi\right) \int_{0}^{1} \frac{d s}{\phi+s\left(\hat{\phi}^{(k)}-\phi\right)}=1_{A}\left(\frac{\hat{\phi}^{(k)}-\phi}{\phi}+\frac{\left(\hat{\phi}^{(k)}-\phi\right)^{2}}{\phi} \int_{0}^{1} \frac{-s d s}{s \hat{\phi}^{(k)}+(1-s) \phi}\right)$.

On $A, s \hat{\phi}^{(k)}+(1-s) \phi \geq s k / \sqrt{T}$. Therefore,

$$
\left|T_{1}\right| \leq \frac{\left|\hat{\phi}^{(k)}-\phi\right|}{\phi}+\frac{\left(\hat{\phi}^{(k)}-\phi\right)^{2}}{\phi} \frac{\sqrt{T}}{k} .
$$

So,

$$
\mathbb{E}\left(T_{1}^{2} \mid \phi\right) \leq \frac{2}{\phi^{2}}\left(\mathbb{E}\left(\left(\hat{\phi}^{(k)}-\phi\right)^{2} \mid \phi\right)+\frac{T}{k^{2}} \mathbb{E}\left(\left(\hat{\phi}^{(k)}-\phi\right)^{4} \mid \phi\right)\right) .
$$

By Corollary 3.3 with both $\gamma=1$ and $\gamma=2$,

$$
\mathbb{E}\left(T_{1}^{2} \mid \phi\right) \lesssim \frac{1}{T \phi^{2}}\left(1+\phi+\phi^{4}+\phi^{-2}+\phi^{-3}+\phi^{2}+\phi^{8}+\phi^{-4}+\phi^{-6}\right) .
$$

Thus, provided that $a>8, \mathbb{E}\left(T_{1}^{2}\right) \lesssim \frac{1}{T}$.

Next, we study $T_{2}$ :

$$
\begin{aligned}
\mathbb{P}\left(A^{c} \mid \phi\right) & \leq \mathbb{P}\left(\hat{\phi}<\frac{k}{\sqrt{T}} \mid \phi\right)+\mathbb{P}\left(V_{T} / T<\frac{k}{\sqrt{T}} \mid \phi\right) \leq \mathbb{P}\left(\hat{\phi}<\frac{k}{\sqrt{T}}, V_{T} / T \geq \frac{k}{\sqrt{T}} \mid \phi\right)+2 \mathbb{P}\left(V_{T} / T<\frac{k}{\sqrt{T}} \mid \phi\right) \\
& \leq \mathbb{P}\left(\hat{\phi}^{(k)}<\frac{k}{\sqrt{T}} \mid \phi\right)+2 \mathbb{P}\left(V_{T} / T<\frac{k}{\sqrt{T}} \mid \phi\right) .
\end{aligned}
$$

The bound for the second term above is given in (3.20): For all $p \geq 1$,

$$
\mathbb{P}\left(V_{T} / T<k / \sqrt{T} \mid \phi\right) \lesssim T^{-p}\left(\phi^{2 p}+\phi^{-2 p}+\phi^{-p}\right) .
$$

Next, as $(\phi-k / \sqrt{T} \geq \phi / 2)=(\phi \geq 2 k / \sqrt{T})$, we have the bound for all $\gamma, s \geq 1$

$$
\begin{aligned}
\mathbb{P}\left(\hat{\phi}^{(k)}<\frac{k}{\sqrt{T}} \mid \phi\right) & \leq \mathbb{P}\left(\left|\phi-\hat{\phi}^{(k)}\right| \geq \frac{\phi}{2}\right)+1_{\left(\phi^{-1} \geq \frac{\sqrt{T}}{2 k}\right)} \\
& \leq\left(\frac{2}{\phi}\right)^{2 \gamma} \mathbb{E}\left(\left|\hat{\phi}^{(k)}-\phi\right|^{2 \gamma} \mid \phi\right)+\left(\frac{2 k}{\sqrt{T}}\right)^{2 s} \phi^{-2 s} .
\end{aligned}
$$

Finally, taking $\gamma=p=s=1$,

$$
\mathbb{E}\left(T_{2}^{2} \mid \phi\right) \lesssim T^{-1}(\log \phi)^{2}\left(\phi^{-1}+\phi^{-2}+\phi^{2}+\phi^{-4}+\phi^{-5}\right) .
$$

Provided that $a>5, \mathbb{E}\left(T_{2}^{2}\right) \lesssim T^{-1}$. The Lemma follows from the two inequalities for $T_{1}$ and $T_{2}$.

The proof of Proposition 4.1 is now complete. 


\section{REFERENCES}

Comte, F., Genon-Catalot, V. and Samson, A. (2013). Nonparametric estimation for stochastic differential equations with random effects. Stoch. Proc. and Appl. 123, 2522-2551.

Davidian, M. and Giltinan, D. (1995). Non linear models to repeated measurement data. Chapman and Hall.

Delattre, M., Genon-Catalot, V. and Samson, A. (2012). Maximum likelihood estimation for stochastic differential equations with random effects. Scand. J. Statist. 40, 322-343.

Delattre, M., Genon-Catalot, V. and Samson, A. (2014). Estimation of population parameters in stochastic differential equations with random effects in the diffusion coefficient. Preprint MAP5 2014-07.

Dion, C. (2014). Nonparametric estimation in a mixed-effect Ornstein-Uhlenbeck model. Preprint MAP5 2014.

Dion, C. and Genon-Catalot, V. (2015). Bidimensional random effect estimation in mixed stochastic differential model. Stat. Inference Stoch. Process, DOI 10.1007/s11203-015-9122-0.

Ditlevsen, S. and De Gaetano, A. (2005). Mixed effects in stochastic differential equation models. Statistical Journal 3, 137-153.

Donnet, S. and Samson, A. (2008). Parametric inference for mixed models defined by stochastic differential equations. ESAIM P \& S 12, 196-218.

Genon-Catalot, V., Jeantheau, T. and Larédo, C. (2000). Stochastic volatility models as hidden Markov models and statistical applications. Bernoulli 6, 1051-1079.

Hall, P. and Heyde, C.C. (1980). Martingale limit theory and its application. Academic press.

Karatzas, I. and Shreve, S.E. (2000). Brownian motion and stochastic calculus. Second edition. Springer-Verlag, New-York.

Kessler, M., Lindner, A. and Sorensen, M. (editors) (2012). Statistical methods for stochastic differential equations. CRC Press. Chapman \& Hall.

Kuhn, E. and Lavielle, M. (2004). Coupling an approximation version of em with an mcmc procedure. ESAIM P \& S 8, 115-131.

Kutoyants, Y.A. (2004). Statistical inference for ergodic diffusion processes. Springer Verlag.

Maitra, T. and Bhattacharya, S. (2014). On asymptotics related to classical inference in stochastic differential equations with random effects. arXiv:140\%.3968v1. 1-12.

Nie, L. (2006).Strong consistency of the maximum likelihood estimator in generalizedlinear and nonlinear mixed-effects models. Metrika 63,123-143.

Nie, L. (2007). Convergence rate of the MLE in generalized linear and nonlinear mixed-effects models: theory and applications. Journal of Statistical Planning and Inference 137, 17871804.

Nie, L. and Yang, M. (2005). Strong consistency of the MLE in nonlinear mixed-effects models with large cluster size. Sankhya: The Indian Journal of Statistics 67, 736-763.

Overgaard, R.V., Jonsson, N., Tornoe, W. and Madsen, H.(2005). Non-linear Mixed-effects models with stochastic differential equations: implementation of an estimation algorithm. Journal of Pharmacokinetics and Pharmacodynamics 32, 85-107.

Picchini, U., De Gaetano, A. and Ditlevsen, S. (2010). Stochastic differential mixed-effects models. Scand. J. Statist. 37, 67-90.

Picchini, U. and Ditlevsen, S. (2011). Practical estimation of high dimensional stochastic differential mixed-effects models. Computational Statistics $\&$ Data Analysis 55, 1426-1444.

Pinheiro, J.C. and Bates, D.M. (2000). Mixed-Effects Models in $S$ and S-PLUS. New York, Springer. 
Corresponding author: C. Larédo, e-mail: catherine.laredo@jouy.inra.fr 